$\underline{\text { Research Article }}$

\title{
Aetiologies and therapies of nodding syndrome: a systematic review and meta-analysis
}

\author{
Helen J Stacey ${ }^{1}$, Lily Woodhouse ${ }^{2}$, Susan C Welburn², Joshua D Jones ${ }^{2}$ \\ 1 University of Edinburgh, Edinburgh Medical School, University of Edinburgh, Edinburgh, UK, 2 University of Edinburgh, Division of Infection and \\ Pathway Medicine, Edinburgh Medical School: Biomedical Sciences, Edinburgh, UK \\ Keywords: global health \\ https://doi.org/10.29392/joghr.3.e2019066
}

\section{Journal of Global Health Reports}

Vol. 3, 2019

\section{Background}

Nodding syndrome (NS) is a childhood epileptic disorder of unknown aetiology confined to parts of South Sudan, Uganda and the United Republic of Tanzania. Patients suffer from atonic seizures that result in characteristic head nodding, and may develop other neurological, endocrinological and psychiatric manifestations. The objective of this study was to adopt a non-judgemental approach to review the hypothesised aetiologies and therapeutic options available for NS. To explore the reported association between NS and Onchocerca volvulus (OV) a meta-analysis of the prevalence of OV among NS cases and controls was performed.

\section{Methods}

Systematic searching of five electronic databases yielded 26 articles that addressed potential aetiologies of NS and 12 that explored the treatment of NS.

\section{Results}

Nine articles contained data about the prevalence of OV in NS cases that was suitable for inclusion in the meta-analysis. Four broad aetiological themes were identified. 1) Nutritional associations included biomarkers of malnutrition and conflicting reports regarding the consumption of some foodstuffs. 2) Regional conflict and the associated psychological trauma have led to consideration of psychiatric aetiologies. 3) There is a strong epidemiological association between NS and Onchocerca volvulus. The meta-analysis in this study revealed that $68.0 \%$ (95\% confidence interval $(\mathrm{CI})=45.2-87.0)$ of 416 NS patients were OV+, compared to $46.6 \%$ (95\% CI=16.4-78.1) of 321 controls. Heterogeneity among both cases $\left(I^{2}=95.5 \%, 95 \% \mathrm{CI}=93.3-97.0\right)$ and controls $\left(I^{2}=97.1 \%\right.$, $95 \% \mathrm{CI}=95.5-98.2)$ was high. The difference of $21.4 \%$ in OV prevalence between the pooled populations was significant $(P<0.0001)$. It is thought that NS represents a form of onchocerciasis-associated epilepsy, however there are also reported associations with Mansonella pestans and measles virus. 4) Autoantibodies against leiomodin-1 and the voltage-gated potassium channel have been associated with NS. Molecular mimicry between leiomodin-1 and $O$. volvulus tropomyosin is a recently suggested aetiology. Without a clear aetiology treatment is symptomatic and patients have been shown to improve with a combination of anti-epileptic medication and supportive therapy.

\section{Conclusions}

The aetiology of NS remains unclear. The leading theory is that NS represents a form of onchocerciasis-associated epilepsy secondary to autoimmunity driven by molecular mimicry. Alternatively, it has been suggested that NS may represent a post-infectious sequelae of measles virus infection. However, each theory currently leaves some questions unanswered. It remains important to understand the aetiology of NS to better prevent or treat future outbreaks.

Nodding syndrome (NS) is a neurological condition of unknown aetiology confined to parts of the United Republic of Tanzania (URT), South Sudan and Uganda. The characteristic head nodding that gives the syndrome its name was first recorded as a symptom of epilepsy in the URT in 1965 and retrospective analysis has found evidence for possible cases as early as $1934 .^{1,2}$ However, it was not until 
Table 1. Nodding syndrome case definition criteria. Adapted from Idro et al. ${ }^{5}$

\begin{tabular}{|c|c|}
\hline A suspected case: & $\begin{array}{l}\text { Reported head nodding in a previously normal person; head nodding was defined as } \\
\text { repetitive, involuntary drops of the head to the chest on two or more occasions. This case } \\
\text { definition is used at the community level. }\end{array}$ \\
\hline \multirow{10}{*}{$\begin{array}{l}\text { A probable case is a suspected } \\
\text { case with: }\end{array}$} & Both of the following major criteria: \\
\hline & -Age at onset of nodding between 3 and 18 years old \\
\hline & -Frequency of nodding 5 to 20 per minute \\
\hline & Plus at least one of the following minor criteria: \\
\hline & $\begin{array}{l}\text {-Other neurological abnormalities (cognitive decline, school dropout due to cognitive/ } \\
\text { behavioural problems, other seizures or neurological abnormalities) }\end{array}$ \\
\hline & -Clustering in space or time with similar cases \\
\hline & -Triggered by food and/or cold weather \\
\hline & -Stunting or wasting \\
\hline & -Delayed sexual or physical development \\
\hline & -Psychiatric symptoms \\
\hline $\begin{array}{l}\text { A confirmed case is a probable } \\
\text { case plus a documented } \\
\text { nodding episode that is: }\end{array}$ & Observed by a trained healthcare worker or recorded on film or on EEG/EMG. \\
\hline
\end{tabular}

epidemics of NS were later recorded in South Sudan and Uganda in the 1990s and 2000s that NS attracted attention.

NS primarily affects children aged 5 to $15 .{ }^{3}$ Patients experience progressive neurological, and in some cases endocrinological, dysfunction; case definition criteria are shown in table $1 .{ }^{4}$ The extent of mortality directly associated with NS remains unclear. Anecdotal evidence has suggested that the greatest risk to NS patients may come from incidental deaths occurring during seizures. ${ }^{4}$ Seizures are initially atonic although other forms, including myoclonic, absence and generalised tonic-clonic seizures can manifest at later stages of the disease. ${ }^{5}$

A range of possible aetiologies have been suggested for NS, including associations with diet and nutrition, Onchocera volvulus (OV), Mansonella pestans, measles virus and autoantibodies as well as psychogenic causes.4,6-8 The most recent hypothesis is that NS may represent an autoimmune consequence of molecular mimicry between the autoantigen leiomodin-1 (LM-1) and OV tropomyosin. ${ }^{8}$ Despite the lack of a definitive aetiology, the term 'onchocerciasis-associated epilepsy' (OAE) has emerged in the literature. ${ }^{9}$ OAE refers to NS alongside other forms of epilepsy with postulated links to OV infection. These include Nakalanga syndrome, documented in Uganda, Burundi and Ethiopia, and other forms of epilepsy, some of which have been documented with head nodding but not confirmed as cases of NS. ${ }^{10,11}$ There is increasing evidence for $\mathrm{OAE}$, with a recent prospective study demonstrating an association between skin microfilarial density and later risk of seizures or epilepsy. ${ }^{12}$ With the exception of a syndrome described in Mexico in 1938, ${ }^{13}$ OAE has thus far only been identified in Africa. No NS cases have been reported beyond the URT, South Sudan or Uganda and no new epidemic cases have been recorded since 2013. ${ }^{14}$

Without a defined aetiology, therapeutic approaches to NS have been forced to take a symptomatic approach, with guidelines published in 2013.5 A comprehensive programme of pharmacological, physical, speech, language, nutritional and mental health therapy has been shown to improve the condition of NS patients. ${ }^{15}$

Given recent developments regarding the potential aetiology of $\mathrm{NS},{ }^{8}$ and a shift towards the phrase OAE in the literature, a systematic review and critique of the proposed aetiologies and therapies of NS and a meta-analysis of the prevalence of OV among NS cases compared with controls is warranted. To maximise the evidence retrieved for nonjudgemental appraisal the systematic searches in this review were conducted without restriction on intervention, comparison or study design.

\section{METHODS}

Systematic searches were conducted on 21 June 2018 using five electronic databases: EMBASE (1980-2018), Ovid MEDLINE In-Process \& Other Non-Indexed Citations and Ovid MEDLINE ${ }^{\circledR}$ (1946-2018), Global Health (1973-2018), Web of Science and BIOSIS Citation Index (1926-2018). The Web of Science Core Collection Citation Indexes searched were: Science Citation Index Expanded (1900-2018), Conference Proceedings Citation Index- Science (1990-2018), Book Citation Index- Science (2005-2018) and the Emerging Sources Citation Index (2015-2018). Each database was searched using the terms 'nodding syndrome' OR 'nodding disease' OR 'luc?luc', without restriction on language, study date or design. For databases that used the Ovid platform (MEDLINE, EMBASE and Global Health) each of these terms were followed by the suffix '.ti,ab.', instigating a search of the title and abstract. The term 'nodding syndrome' was also searched as a medical subject heading. For databases accessed through the Web of Science platform, each of the three terms above were searched as topics. Additional manuscripts were identified using manual searching. ${ }^{16-20}$

\section{SELECTION OF STUDIES}

Deduplication was performed using Endnote (version X8.0.1) and Zotero (version 5.0.47). All studies underwent 
title and abstract screening. Studies were deemed eligible if they were available in English and explicitly addressed NS. Studies which addressed the broader field of OAE and did not explicitly examine NS were excluded. Studies deemed eligible by title and abstract screen were accessed in fulltext. Presentation abstracts were deemed to exist as fulltext. Thereafter articles not containing primary data regarding the aetiology or treatment of NS were excluded. Studies which could not be accessed in full-text and did not state sufficient information in the abstract were excluded. Studies reporting primary data reported elsewhere were excluded. Both title and abstract and full-text screening were performed independently by two authors (JDJ, HJS), with discrepancies resolved by agreement. To minimise bias, studies eligible for the meta-analysis contained data from which the prevalence of OV in $\geqslant 10$ NS patients or, if available, $\geqslant 10$ controls could be calculated. This review was conducted in accordance with the Preferred Reporting Items for Systematic Reviews and Meta-Analyses (PRISMA) guidelines, ${ }^{21}$ and a PRISMA checklist completed (Appendix S1 in the Online Supplementary Document). A study protocol was not published prior to this study.

\section{DATA EXTRACTION AND RISK OF BIAS}

The following information was extracted from each eligible study: author(s), publication date and title, journal name, study location, date, design and case/control population sizes, key findings and prevalence data for meta-analysis if available. The risk of bias was assessed using the appropriate critical appraisal tool from the suite offered by the Joanna Briggs Institute. ${ }^{22}$ Pre-study randomised controlled trial (RCT) protocols were not subject to critical appraisal.

\section{STATISTICAL ANALYSIS}

A random-effects meta-analysis was performed to calculate the pooled prevalence (with 95\% confidence intervals (Cis)) of OV microfilariae in skin snips taken from NS patients compared to controls. Study heterogeneity was assessed by the $\mathrm{I}^{2}$ statistic, reported with $95 \%$ CIs, and interpreted as low ( $\leqslant 25 \%)$, moderate $(25-75 \%)$ and high $(\geqslant 75 \%) .{ }^{23}$ All meta-analyses were conducted using the 'meta-analysis of proportions' function on MedCalc version 18.0 (MedCalc Software, Ostend, Belgium) which uses an arcsine square root transformation to calculate the weighted summary proportion. ${ }^{24}$ The significance of proportions was compared using the MedCalc $\mathrm{N}-1 \chi^{2}$ calculator.

\section{RESULTS}

\section{STUDY SELECTION AND CHARACTERISTICS}

Figure 1 illustrates the study selection process. The systematic search yielded 377 articles; five additional articles were identified from review articles and manual searches. ${ }^{16-20}$ The titles and abstracts of the 161 articles that remained following deduplication were screened, and a further 40 articles excluded. Of the remaining 121 articles accessed in full-text, 82 were excluded because: they were not primary literature $(n=42)$, the full-text was inaccessible $(n=21)$ or they did not contain relevant $(n=16)$ or new $(n=3)$ data. The final 39 studies included 26 that explored potential aetiologies of NS and 13 that explored treatment of NS. Nine studies contained data suitable for meta-analysis of the prevalence of OV among NS cases and controls. These studies formed the core of the review and were supplemented by wider primary and secondary literature.

The selected articles ranged in date from 2008 to 2018 and included studies from South Sudan $(n=4)$, Uganda $(n=22)$, the URT $(n=5)$, the Democratic Republic of Congo $(n=1)$ and the United States of America $(n=1)$. Study details and key findings are shown in Table 2 and Table 3 for aetiological and therapeutic perspectives respectively. The outcome of critical appraisal of the included studies and the list of studies that were excluded are included in Appendices S2 and S3 in the Online Supplementary Document respectively. Critical appraisal revealed a range of shortcomings in reporting across the eligible studies which, while important to highlight, were not considered to indicate bias sufficient to warrant exclusion.

\section{THE AETIOLOGY OF NS}

Appraisal of the data extracted from the 26 articles which investigated theoretical aetiologies of $\mathrm{NS}^{7,8,16,17,25-44}$ revealed four broad aetiological themes which form the basis of this review: metabolic, infectious, autoimmune and psychiatric associations.

\section{METABOLIC}

Many of the populations where a high incidence of NS was recorded had experienced recent conflict and food shortages and often became reliant on relief food. Aid deliveries were regularly disrupted, sometimes resulting in the delivery of mouldy food, often consumed nonetheless. A correlation was identified between the peaks in NS cases and upsurges in conflict and food shortages, with a temporal disparity of 5-7 years. ${ }^{35}$ Moreover, it has been reported that there were no cases of NS in Ugandan conflict zones in which people had not been moved into internally displaced persons (IDP) camps, but instead cases appeared after the establishment of the IDP camps, supplied by relief food. This led to the hypothesis that the rise in NS cases may have been linked to the consumption of specific foodstuffs regularly supplied in the IDP camps. ${ }^{16,17,40}$

Four case-control studies have provided mixed conclusions regarding the association between consumption of (spoiled) relief foods and NS.6,7,36,41 Tumwine and colleagues were the first to suggest an association with food, specifically serena, a type of red/brown sorghum. ${ }^{7}$ However, further analysis of these data confirmed no association with cassava consumption. ${ }^{40}$ Later, Spencer and colleagues found positive associations with the consumption of maize, mouldy maize or relief food. ${ }^{41}$ Obol and colleagues also subsequently identified an association with the consumption of relief food. ${ }^{36}$ However, it has been suggested that recall bias over approximately eight years, and the attribution of blame by those affected to the Ugandan government and international organisations, may have influenced the results obtained by Spencer and colleagues. ${ }^{45}$ In contrast, Foltz and colleagues identified an association with the 
Table 2. Studies examining the aetiology of nodding syndrome

\begin{tabular}{|c|c|c|c|c|c|c|c|c|c|}
\hline Citation & $\begin{array}{l}\text { Publication } \\
\text { date }\end{array}$ & Title & Study location & Study date & Study design & Cases & Controls & Key finding(s) & Journal \\
\hline 43 & 2008 & $\begin{array}{l}\text { The head nodding } \\
\text { syndrome - clinical } \\
\text { classification and } \\
\text { possible causes of a } \\
\text { new epilepsy } \\
\text { disorder }\end{array}$ & Tanzania & N/A & Cohort & 62 & - & $\begin{array}{l}90.3 \% \text { had at at least one } \\
\text { relative with epilepsy. } \\
\text { Association with OV: } 31 / 51 \text { SS } \\
\text { and } 12 / 20 \text { PCRs were OV+. } \\
\text { CSF PCR negative (0/48). }\end{array}$ & Neurology \\
\hline 26 & 2012 & $\begin{array}{l}\text { Nodding syndrome - } \\
\text { South Sudan }\end{array}$ & South Sudan & 2010 & Case-control & 38 & 38 & $\begin{array}{l}\text { NS associated with OV+SS. } \\
\text { Total study figues included } \\
\text { Maridi and Witto } \\
\text { communities. Among the } 25 \\
\text { Maridi matched pairs, } 88 \% \text { of } \\
\text { cases vs } 44 \% \text { of controls were } \\
\text { OV+. No significant OV } \\
\text { association between the } 13 \\
\text { Witto pairs. }\end{array}$ & $\begin{array}{l}\text { Morbidity and } \\
\text { Mortality Weekly } \\
\text { Report }\end{array}$ \\
\hline 7 & 2012 & $\begin{array}{l}\text { Clinical and } \\
\text { epidemiologic } \\
\text { characteristics of } \\
\text { nodding syndrome in } \\
\text { Mundri County, } \\
\text { southern Sudan }\end{array}$ & South Sudan & 2001-02 & Case-control & 82 & 81 & $\begin{array}{l}\text { No association with MV: } 2 / 13 \\
\text { NS patients had a history of } \\
\text { MV vs } 11 / 19 \text { controls. } \\
\text { Association with OV: } 76 / 82 \\
\text { NS patients had OV+ SS vs } 39 / \\
81 \text { controls. HN triggered by } \\
\text { food ( } 73 \% \text { of } 39 \text { cases). } \\
\text { Association with serena } \\
\text { (sorghum): OR=6.22; } P=0.049 \text {. } \\
\text { Association with MP: } \\
\text { OR=3.22; } P=0.05 \text {. }\end{array}$ & $\begin{array}{l}\text { African Health } \\
\text { Sciences }\end{array}$ \\
\hline 6 & 2013 & $\begin{array}{l}\text { An epidemiologic } \\
\text { investigation of } \\
\text { potential risk factors } \\
\text { for nodding } \\
\text { syndrome in Kitgum } \\
\text { District, Uganda }\end{array}$ & Uganda & 2009 & Case-control & 49 & 49 & $\begin{array}{l}\text { No association with MV or } \\
\text { sorghum. Serological } \\
\text { association with OV, exposure } \\
\text { to munitions (AOR=13.9 } \\
\text { [1.4-135.5]), consumption of } \\
\text { crushed cassava roots } \\
\text { (AOR=5.4 [1.3-22.1]), visual } \\
\text { hallucinations (AOR=13.6 } \\
\text { [1.5-121.7]) and vitamin B6 } \\
\text { deficiency (84\% cases, } 75 \% \\
\text { controls). Matched seological } \\
\text { AORs for Ov16 and OvFAR/ } \\
\text { MSA were } 3.16 \text { (0.84-11.89) } \\
\text { and } 20.80(1.37-316.24)\end{array}$ & PLoS One \\
\hline
\end{tabular}




\begin{tabular}{|c|c|c|c|c|c|c|c|c|c|}
\hline Citation & $\begin{array}{l}\text { Publication } \\
\text { date }\end{array}$ & Title & Study location & Study date & Study design & Cases & Controls & Key finding(s) & Journal \\
\hline & & & & & & & & respectively. & \\
\hline 29 & 2013 & $\begin{array}{l}\text { Nodding syndrome in } \\
\text { Ugandan children- } \\
\text { clinical features, } \\
\text { brain imaging and } \\
\text { complications: a case } \\
\text { series }\end{array}$ & Uganda & 2012 & Case series & 22 & - & $\begin{array}{l}\text { MRI and EEG findings } \\
\text { described. } 12 / 22 \text { NS patients } \\
\text { had psychiatric morbidities. A } \\
57 \% \text { reduction in total seizure } \\
\text { burden was achieved with } \\
\text { sodium valproate. }\end{array}$ & BMJ Open \\
\hline 33 & 2013 & $\begin{array}{l}\text { Nodding syndrome } \\
\text { (NS) in Northern } \\
\text { Uganda: a probable } \\
\text { metabolic disorder }\end{array}$ & Uganda & 2012 & Case-control & 101 & 101 & $\begin{array}{l}\text { NS associated with } \\
\text { malnutrition, OV and a high } \\
\text { anion gap. }\end{array}$ & $\begin{array}{l}\text { British Journal of } \\
\text { Medicine and } \\
\text { Medical Research }\end{array}$ \\
\hline 38 & 2013 & $\begin{array}{l}\text { Clinical, neurological, } \\
\text { and } \\
\text { electrophysiological } \\
\text { features of nodding } \\
\text { syndrome in Kitgum, } \\
\text { Uganda: an } \\
\text { observational case } \\
\text { series }\end{array}$ & Uganda & 2009 & Case series & 23 & - & $\begin{array}{l}\text { MRI, CSF and EEG findings } \\
\text { described. No evidence of } \\
\text { inflammation found. }\end{array}$ & Lancet Neurology \\
\hline 2 & 2013 & $\begin{array}{l}\text { Nodding syndrome: } \\
\text { origins and natural } \\
\text { history of a } \\
\text { longstanding } \\
\text { epileptic disorder in } \\
\text { sub-Saharan Africa }\end{array}$ & Tanzania & $1960-71$ & Retrospective & 150 & - & $\begin{array}{l}\text { Historical evidence for } \\
\text { probable NS cases with onset } \\
\text { between } 1934 \text { and } 1962 .\end{array}$ & $\begin{array}{l}\text { African Health } \\
\text { Sciences }\end{array}$ \\
\hline 40 & 2013 & $\begin{array}{l}\text { Nodding syndrome in } \\
\text { Mundri county, South } \\
\text { Sudan: } \\
\text { environmental, } \\
\text { nutritional and } \\
\text { infectious factors }\end{array}$ & South Sudan & $\begin{array}{l}2001 / \\
2012\end{array}$ & Case-control & & & $\begin{array}{l}\text { Analysis of data from Tumwine } \\
\text { and colleagues } 2012 \text {. Adds no } \\
\text { association with cassava } \\
\text { consumption. }\end{array}$ & $\begin{array}{l}\text { African Health } \\
\text { Sciences }\end{array}$ \\
\hline 44 & 2013 & $\begin{array}{l}\text { MRI findings in } \\
\text { people with epilepsy } \\
\text { and nodding } \\
\text { syndrome in an area } \\
\text { endemic for } \\
\text { onchocerciasis: an } \\
\text { observational study }\end{array}$ & Tanzania & N/A & Cohort & 12 & 20 & $\begin{array}{l}\text { MRI findings described. } \\
\text { Cerebral pathology associated } \\
\text { with OV (SS/PCR). Control } \\
\text { group was OCE patients. }\end{array}$ & $\begin{array}{l}\text { African Health } \\
\text { Sciences }\end{array}$ \\
\hline 27 & 2014 & $\begin{array}{l}\text { Nodding syndrome in } \\
\text { Tanzania may not be }\end{array}$ & Tanzania & N/A & Case-control & 22 & 8 & $\begin{array}{l}\text { Controls }=7 \text { healthy, } 1 \text { OCE. No } \\
\text { evidence for NMDA or VKGC }\end{array}$ & $\begin{array}{l}\text { African Health } \\
\text { Sciences }\end{array}$ \\
\hline
\end{tabular}




\begin{tabular}{|c|c|c|c|c|c|c|c|c|c|}
\hline Citation & $\begin{array}{l}\text { Publication } \\
\text { date }\end{array}$ & Title & Study location & Study date & Study design & Cases & Controls & Key finding(s) & Journal \\
\hline & & $\begin{array}{l}\text { associated with } \\
\text { circulating anti- } \\
\text { NMDA- and anti- } \\
\text { VGKC receptor } \\
\text { antibodies or } \\
\text { decreased pyridoxal } \\
\text { phosphate serum } \\
\text { levels-a pilot study }\end{array}$ & & & & & & $\begin{array}{l}\text { autoantibodies. No association } \\
\text { with pyridoxal-phosphate } \\
\text { serum levels. }\end{array}$ & \\
\hline 31 & 2014 & $\begin{array}{l}\text { Detection of auto- } \\
\text { antibodies to } \\
\text { leiomodin-1 in } \\
\text { patients with } \\
\text { nodding syndrome }\end{array}$ & N/A & N/A & Case-control & 19 & 19 & $\begin{array}{l}\text { Anti-LM-1 Ig was detected in } \\
\text { the serum of } 11 / 19 \text { NS } \\
\text { patients and } 5 / 19 \text { controls. } \\
\text { Anti-LM-1 identified in the } \\
\text { CSF of an unreported number } \\
\text { of NS patients, shown to be } \\
\text { neurotoxic in vitro and cross- } \\
\text { react with OV tropomyosin. } \\
\text { LM-1 expression shown in } \\
\text { CNS regions comparable with } \\
\text { reported MRI findings. }\end{array}$ & $\begin{array}{l}\text { Journal of } \\
\text { Neuroimmunology }\end{array}$ \\
\hline 34 & 2014 & $\begin{array}{l}\text { High anion gap } \\
\text { metabolic acidosis } \\
\text { among children with } \\
\text { nodding syndrome } \\
\text { (NS) in Northern } \\
\text { Uganda: case series }\end{array}$ & Uganda & 2012 & Case series & 10 & - & $\begin{array}{l}\text { Of the } 10 \text { probable NS cases } \\
\text { all had hypocalcaemia, raised } \\
\text { bicarbonate and a high anion } \\
\text { gap suggestive of metabolic } \\
\text { acidosis. }\end{array}$ & $\begin{array}{l}\text { British Journal of } \\
\text { Medicine and } \\
\text { Medical Research }\end{array}$ \\
\hline 35 & 2014 & $\begin{array}{l}\text { Nodding syndrome in } \\
\text { Kitgum District, } \\
\text { Uganda: association } \\
\text { with conflict and } \\
\text { internal } \\
\text { displacement }\end{array}$ & N/A & N/A & Retrospective & N/A & N/A & $\begin{array}{l}\text { Retrospective analyses } \\
\text { suggested that conflict and } \\
\text { movement of people into IDP } \\
\text { camps were associated with } \\
\text { rises in NS 5-6 and } 7 \text { years } \\
\text { later respectively. }\end{array}$ & BMJ Open \\
\hline 37 & 2014 & $\begin{array}{l}\text { Physical growth, } \\
\text { puberty and } \\
\text { hormones in } \\
\text { adolescents with } \\
\text { nodding syndrome; a } \\
\text { pilot study }\end{array}$ & Uganda & N/A & $\begin{array}{l}\text { Cross- } \\
\text { sectional }\end{array}$ & 10 & - & $\begin{array}{l}\text { Endocrinological analysis } \\
\text { suggestive of pituitary growth } \\
\text { and gonadal hormone axis } \\
\text { dysfunction. } 5 / 8 \text { NS patients } \\
\text { had stunting, } 8 / 8 \text { had a normal } \\
\text { serum GH level, but } 3 / 8 \text { had } \\
\text { low IGF1/IGFBP3. Thyroid, } \\
\text { adrenal, parathyroid, prolactin } \\
\text { levels were normal. }\end{array}$ & $\begin{array}{l}\text { BMC Research } \\
\text { Notes }\end{array}$ \\
\hline 32 & 2015 & Nodding episodes & Uganda & N/A & Case report & 1 & - & Report of a 13-year-old male & British Journal of \\
\hline
\end{tabular}




\begin{tabular}{|c|c|c|c|c|c|c|c|c|c|}
\hline Citation & $\begin{array}{l}\text { Publication } \\
\text { date }\end{array}$ & Title & Study location & Study date & Study design & Cases & Controls & Key finding(s) & Journal \\
\hline & & $\begin{array}{l}\text { and high anion gap in } \\
\text { a 13-year-old child } \\
\text { with nodding } \\
\text { syndrome: a case } \\
\text { report }\end{array}$ & & & & & & $\begin{array}{l}\text { with probable NS and } \\
\text { pyomystitis, as reported in } \\
\text { Kitara 2013. Adds temporal } \\
\text { association between an } \\
\text { elevated anion gap and the } \\
\text { presence of seizures. }\end{array}$ & $\begin{array}{l}\text { Medicine and } \\
\text { Medical Research }\end{array}$ \\
\hline 39 & 2015 & $\begin{array}{l}\text { Nodding syndrome: } \\
\text { an epileptic disorder } \\
\text { restricted to Africa? }\end{array}$ & N/A & 2014 & Case-control & 50 & 50 & $\begin{array}{l}\text { Much data available in } \\
\text { Spencer and colleagues } 2016 . \\
\text { This adds that mycotoxin } \\
\text { analysis of plasma and urine } \\
\text { found no difference between } \\
\text { cases and controls. }\end{array}$ & $\begin{array}{l}\text { Journal of the } \\
\text { Neurological } \\
\text { Sciences }\end{array}$ \\
\hline 30 & 2016 & $\begin{array}{l}\text { Is nodding syndrome } \\
\text { an Onchocerca } \\
\text { volvulus-induced } \\
\text { neuroinflammatory } \\
\text { disorder? Uganda's } \\
\text { story of research in } \\
\text { understanding the } \\
\text { disease }\end{array}$ & N/A & 2013 & Case-control & 31 & 11 & $\begin{array}{l}\text { Review, included as it contains } \\
\text { data not published elsewhere. } \\
15 / 31 \text { NS patients vs } 1 / 11 \\
\text { controls were anti-VGKC Ig } \\
\text { positive. }\end{array}$ & $\begin{array}{l}\text { International } \\
\text { Journal of } \\
\text { Infectious } \\
\text { Diseases }\end{array}$ \\
\hline 36 & 2016 & $\begin{array}{l}\text { Reduced plasma } \\
\text { concentrations of } \\
\text { vitamin B6 and } \\
\text { increased plasma } \\
\text { concentrations of the } \\
\text { neurotoxin } \\
\text { 3-hydroxykynurenine } \\
\text { are associated with } \\
\text { nodding syndrome: a } \\
\text { case control study in } \\
\text { Gulu and Amuru } \\
\text { districts, Northern } \\
\text { Uganda }\end{array}$ & Uganda & 2013 & Case-control & 66 & 73 & $\begin{array}{l}\text { Low plasma vitamin } B 6 \\
(A O R=7.22, P=0.001) \text {, raised } \\
3-H K(A O R=4.50, P=0.0130 \text { ), } \\
\text { child being cared for by } \\
\text { mother only ( } A O R=5.43 \text {, } \\
P=0.011) \text {, child being cared for } \\
\text { by guardian ( } A O R=5.90 \text {, } \\
P=0.019 \text { ) and consumption of } \\
\text { relief food at weaning (AOR= } \\
4.05, P=0.021 \text { ) were } \\
\text { associated with NS. }\end{array}$ & $\begin{array}{l}\text { Pan African } \\
\text { Medical Journal }\end{array}$ \\
\hline 42 & 2016 & $\begin{array}{l}\text { Nodding syndrome: } \\
2015 \text { international } \\
\text { conference report } \\
\text { and Gulu Accord }\end{array}$ & N/A & N/A & Mixed & N/A & N/A & $\begin{array}{l}\text { Report from the } 2015 \\
\text { international conference on } \\
\text { NS. Results presented, not } \\
\text { found elsewhere, include a } \\
\text { case-control study ( } n=39 \text { and } \\
41 \text { respectively) that } \\
\text { associated low houshold } \\
\text { income and not purifying }\end{array}$ & eNeurologicalSci \\
\hline
\end{tabular}




\begin{tabular}{|c|c|c|c|c|c|c|c|c|c|}
\hline Citation & $\begin{array}{l}\text { Publication } \\
\text { date }\end{array}$ & Title & Study location & Study date & Study design & Cases & Controls & Key finding(s) & Journal \\
\hline & & & & & & & & $\begin{array}{l}\text { water with NS. A cross- } \\
\text { sectional study investigating } \\
\text { the psychiatric and } \\
\text { psychological features of } 225 \\
\text { Ugandan NS patients, the } \\
\text { majority of cases ( } 51.6 \% \text { male, } \\
48.4 \% \text { female) started HN in } \\
\text { IDP camps between } 2005-10 \text {. } \\
\text { Psychiatric disorders included } \\
\text { major episodic depression } \\
\text { (25.3\%), PTSD (16.4\%), GAD } \\
\text { (30.7\%) and PDD (4\%). }\end{array}$ & \\
\hline 41 & 2016 & $\begin{array}{l}\text { Environmental, } \\
\text { dietary and case- } \\
\text { control study of } \\
\text { nodding syndrome in } \\
\text { Uganda: a post- } \\
\text { measles brain } \\
\text { disorder triggered by } \\
\text { malnutrition? }\end{array}$ & Uganda & 2014 & Case-control & 50 & 50 & $\begin{array}{l}\text { Onset of NS peaked in } 2003 \\
\text { and } 2008, \text { primarily in April } \\
\text { and June. Eating emergency } \\
\text { food (OR=4.0 [1.3-17.6], } \\
p=0.016), \text { maize }(\mathrm{OR}=4.00 \\
[1.0-26.5]) \text {, mouldy maize } \\
\text { (OR=4.33 [1.4-18.9], } \mathrm{p}=0.009) \\
\text { and a history of } \mathrm{MV}(\mathrm{OR}=6.00 \\
\text { [1.03-113], } \mathrm{p}=0.047) \text { were } \\
\text { associated with NS. }\end{array}$ & $\begin{array}{l}\text { Journal of the } \\
\text { Neurological } \\
\text { Sciences }\end{array}$ \\
\hline 25 & 2017 & $\begin{array}{l}\text { Metabolic analysis of } \\
\text { children affected } \\
\text { with nodding } \\
\text { syndrome in north } \\
\text { Uganda: A pilot study }\end{array}$ & Uganda & 2014 & Cohort & 48 & - & $\begin{array}{l}\text { Much data available in Denis } \\
\text { and colleagues } 2017 \text {. This } \\
\text { study adds clinical } \\
\text { biochemistry findings. } 37 / 47 \\
\text { had low biotinidase. Mean } \\
\text { BMI was 16.9. Mean acetyl } \\
\text { carnitine level was low. } \\
\text { Proprionyl carnitine and } \\
\text { butyryl carnitine levels were } \\
\text { elevated. }\end{array}$ & $\begin{array}{l}\text { Developmental } \\
\text { Medicine and } \\
\text { Child Neurology }\end{array}$ \\
\hline 17 & 2017 & $\begin{array}{l}\text { Is there a line } \\
\text { between internal } \\
\text { displacement; } \\
\text { environmental and } \\
\text { dietary factors in the } \\
\text { onset of nodding } \\
\text { syndrome in } \\
\text { northern Uganda? A } \\
\text { clinical observational } \\
\text { study design }\end{array}$ & Uganda & 2014 & Cohort & 48 & - & $\begin{array}{l}\text { Duration of stay in IDP camps } \\
\text { and being in a family in which } \\
\text { the firstborn had NS were } \\
\text { strongly associated with NS. }\end{array}$ & $\begin{array}{l}\text { World Journal of } \\
\text { Pharmaceutical } \\
\text { and Medical } \\
\text { Research }\end{array}$ \\
\hline
\end{tabular}




\begin{tabular}{|c|c|c|c|c|c|c|c|c|c|}
\hline Citation & $\begin{array}{l}\text { Publication } \\
\text { date }\end{array}$ & Title & Study location & Study date & Study design & Cases & Controls & Key finding(s) & Journal \\
\hline 28 & 2017 & $\begin{array}{l}\text { Nodding syndrome: } \\
\text { multimycotoxin case- } \\
\text { control study in } \\
\text { Northern Uganda }\end{array}$ & Uganda & 2014 & Case-control & 50 & 50 & $\begin{array}{l}\text { Analysis of plasma and urine } \\
\text { samples from Spencer and } \\
\text { colleagues } 2016 . \text { Mycotoxins } \\
\text { were detected in } 14 \% \text { and } \\
12 \% \text { of NS and control cases } \\
\text { respectively; concluded the } \\
\text { mycotoxin burden was similar. }\end{array}$ & $\begin{array}{l}\text { Annals of } \\
\text { Neurology }\end{array}$ \\
\hline 8 & 2017 & $\begin{array}{l}\text { Nodding syndrome } \\
\text { may be an } \\
\text { autoimmune reaction } \\
\text { to the parasitic worm } \\
\text { Onchocerca volvulus }\end{array}$ & $\mathrm{N} / \mathrm{A}$ & N/A & Case-control & 55 & 55 & $\begin{array}{l}\text { Added data for a further } 36 \\
\text { cases and controls to the data } \\
\text { from Johnson } 2014 \text {, giving } 55 \\
\text { matched pairs. Of the NS } \\
\text { cases, } 80 \% \text { were OV+ and } \\
52.7 \% \text { were anti-LM-1 Ig+. Of } \\
\text { the controls, } 45.5 \% \text { were OV+ } \\
\text { and } 30.9 \% \text { were anti-LM-1 Ig+. } \\
\text { The finding that anti-LM-1 Ig } \\
\text { to be neurotoxic in vitro and } \\
\text { cross-react with OV } \\
\text { tropomyosin was confirmed. } \\
\text { LM-1 expression shown in } \\
\text { CNS regions comparable with } \\
\text { reported MRI findings. 8/16 } \\
\text { CSF samples from NS patients } \\
\text { were anti-LM-1 Ig+, compared } \\
\text { to } 0 / 8 \text { North American } \\
\text { controls. }\end{array}$ & $\begin{array}{l}\text { Science } \\
\text { Translational } \\
\text { Medicine }\end{array}$ \\
\hline 16 & 2018 & $\begin{array}{l}\text { Could nodding } \\
\text { syndrome in } \\
\text { Northern Uganda be } \\
\text { a form of autism }\end{array}$ & Uganda & 2014 & Cohort & $?$ & $?$ & $\begin{array}{l}\text { Much data available in Denis } \\
\text { and colleagues } 2017 \text {. This } \\
\text { study conjectures that NS may } \\
\text { be akin to ASD. }\end{array}$ & $\begin{array}{l}\text { Pan African } \\
\text { Medical Journal }\end{array}$ \\
\hline
\end{tabular}

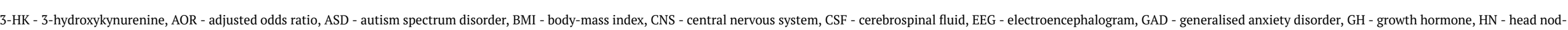

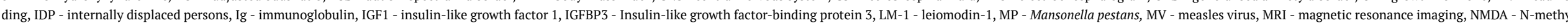

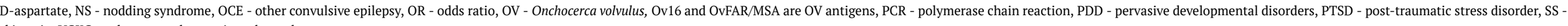
skin snip, VGKC - voltage-gated potassium channel 
Table 3. Studies examining therapeutic options for nodding syndrome

\begin{tabular}{|c|c|c|c|c|c|c|c|c|c|}
\hline Citation & $\begin{array}{l}\text { Publication } \\
\text { date }\end{array}$ & Title & Study location & Study date & Study design & Cases & Controls & Key finding(s) & Journal \\
\hline 75 & 2013 & $\begin{array}{l}\text { Pyomyositis in } \\
\text { Nodding syndrome } \\
\text { (NS) patient--a case } \\
\text { report }\end{array}$ & Uganda & N/A & Case report & 1 & - & $\begin{array}{l}\text { Pyomyostitis in a probable } \\
\text { NS case. Seizures treated } \\
\text { with sodium valproate. }\end{array}$ & $\begin{array}{l}\text { The Pan African } \\
\text { Medical Journal }\end{array}$ \\
\hline 52 & 2013 & $\begin{array}{l}\text { Neuropsychiatric } \\
\text { perspectives on } \\
\text { nodding syndrome } \\
\text { in northern } \\
\text { Uganda: a case } \\
\text { series study and a } \\
\text { review of the } \\
\text { literature }\end{array}$ & Uganda & N/A & Case series & 6 & - & $\begin{array}{l}\text { NS patients may have } \\
\text { histories of psychological } \\
\text { trauma, and comorbidities } \\
\text { including PTSD, DTD and } \\
\text { depression. Treatments } \\
\text { included CBZ or PHB and } \\
\text { sometimes imipramine, } \\
\text { with mixed results. }\end{array}$ & $\begin{array}{l}\text { African Health } \\
\text { Sciences }\end{array}$ \\
\hline 15 & 2014 & $\begin{array}{l}\text { Patients with } \\
\text { nodding syndrome } \\
\text { in Uganda improve } \\
\text { with symptomatic } \\
\text { treatment: a cross- } \\
\text { sectional study }\end{array}$ & Uganda & 2013 & Cohort & 484 & 476 & $\begin{array}{l}\text { Controls were patients } \\
\text { with OCE. Treatment } \\
\text { according to the } 2013 \\
\text { guidelines resulted in } 25 \% \\
\text { of NS patients being } \\
\text { seizure free. }>70 \% \text { had a } \\
\text { reduced seizure frequency. } \\
\text { Emotional and behavioural } \\
\text { difficulties resolved in } \\
59 \% .86 / 484 \text { children who } \\
\text { had not previously been } \\
\text { able to attend school could } \\
\text { now do so. } 83.1 \% \text { achieved } \\
\text { independence in basic self- } \\
\text { care. }\end{array}$ & BMJ Open \\
\hline 76 & 2014 & $\begin{array}{l}\text { A longitudinal } \\
\text { study on nodding } \\
\text { syndrome--a new } \\
\text { African epilepsy } \\
\text { disorder }\end{array}$ & Tanzania & 2009 & Cohort & 62 & - & $\begin{array}{l}\text { Longitudinal follow-up } \\
\text { study on Winkler and } \\
\text { colleagues } 2008 . \mathrm{HN} \\
\text { decreased in } 11 \text {, increased } \\
\text { in } 2 \text {, did not change in } 4 \\
\text { and stopped in } 15 \text { of } 32 \\
\text { patients treated with PHB. } \\
\text { Of the } 15,13 \text { still had } \\
\text { GTCS. HN stopped in } 3 \\
\text { patients treated with CBZ, } \\
\text { all continued to have } \\
\text { GTCS. }\end{array}$ & Epilepsia \\
\hline 78 & 2015 & Catatonia in & Uganda & N/A & Cross- & 33 & - & $16 / 33$ patients met & BMC Research \\
\hline
\end{tabular}




\begin{tabular}{|c|c|c|c|c|c|c|c|c|c|}
\hline Citation & $\begin{array}{l}\text { Publication } \\
\text { date }\end{array}$ & Title & Study location & Study date & Study design & Cases & Controls & Key finding(s) & Journal \\
\hline & & $\begin{array}{l}\text { Ugandan children } \\
\text { with nodding } \\
\text { syndrome and } \\
\text { effects of } \\
\text { treatment with } \\
\text { lorazepam: a pilot } \\
\text { study }\end{array}$ & & & sectional & & & $\begin{array}{l}\text { diagnostic criteria for } \\
\text { catatonia. Lorazepam } \\
\text { administration reduced } \\
\text { the severity of catatonia } \\
\text { symptoms in } 10 \text { of } 16 \\
\text { patients, and by more than } \\
50 \% \text { in } 6 .\end{array}$ & Notes \\
\hline 48 & 2015 & $\begin{array}{l}\text { Evaluation and } \\
\text { immunomodulatory } \\
\text { treatment at the } \\
\text { NIH of children } \\
\text { with nodding } \\
\text { syndrome from } \\
\text { northern Uganda }\end{array}$ & USA & $\mathrm{N} / \mathrm{A}$ & Case series & 3 & - & $\begin{array}{l}\text { Abstract only available. } 3 \\
\text { related NS patients were } \\
\text { examined. CSF from } 1 / 3 \\
\text { had oligoclonal bands, } \\
\text { another had partially } \\
\text { identical bands in CSF and } \\
\text { serum. Biomarkers for } \\
\text { pyridoxine-responsive } \\
\text { seizures were absent in all. } \\
\text { Mt DNA was normal. } 2 / 3 \\
\text { were given } \\
\text { plasmapheresis, 1/3 was } \\
\text { given IVIG, all were given } \\
\text { pyridoxine. No HN } \\
\text { episodes recorded, } 1 / 3 \text { had } \\
\text { multiple seizures. }\end{array}$ & Neurology \\
\hline 77 & 2015 & $\begin{array}{l}\text { Neurophysiological } \\
\text { and clinical findings } \\
\text { on nodding } \\
\text { syndrome in } 21 \\
\text { South Sudanese } \\
\text { children and a } \\
\text { review of the } \\
\text { literature }\end{array}$ & South Sudan & $2012-13$ & Case series & 21 & - & $\begin{array}{l}18 \text { probable cases, } 3 \\
\text { confirmed. Clincial and } \\
\text { EEG findings described. } \\
\text { None achieved good } \\
\text { seizure control with CBZ } \\
\text { monotherapy }(n=9) \text { or in } \\
\text { combination with PHB } \\
(n=9) \text { or PHT }(n=3) \text {. }\end{array}$ & $\begin{array}{l}\text { Seizure- } \\
\text { European } \\
\text { Journal of } \\
\text { Epilepsy }\end{array}$ \\
\hline 11 & 2015 & $\begin{array}{l}\text { Case report: } \\
\text { nodding syndrome, } \\
\text { Western Uganda, } \\
1994\end{array}$ & Uganda & 1994 & Case report & 1 & - & $\begin{array}{l}\text { Description of NS in a } \\
15 \text {-year-old male from an } \\
\text { OV endemic area in an } \\
\text { area of Western Uganda } \\
\text { not previously associated } \\
\text { with NS. Treatment with } \\
\text { PHB did not achieve long- } \\
\text { term seizure control. }\end{array}$ & $\begin{array}{l}\text { American } \\
\text { Journal of } \\
\text { Tropical } \\
\text { Medicine and } \\
\text { Hygiene }\end{array}$ \\
\hline 72 & 2016 & $\begin{array}{l}\text { Health seeking } \\
\text { behaviours among }\end{array}$ & Uganda & 2013 & $\begin{array}{l}\text { Cross- } \\
\text { sectional }\end{array}$ & $\begin{array}{c}249 \\
\text { caregivers }\end{array}$ & - & $\begin{array}{l}78.3 \% \text { of caretakers } \\
\text { sought help from a health }\end{array}$ & PLoS One \\
\hline
\end{tabular}




\begin{tabular}{|c|c|c|c|c|c|c|c|c|c|}
\hline Citation & $\begin{array}{l}\text { Publication } \\
\text { date }\end{array}$ & Title & Study location & Study date & Study design & Cases & Controls & Key finding(s) & Journal \\
\hline & & $\begin{array}{l}\text { caretakers of } \\
\text { children with } \\
\text { nodding syndrome } \\
\text { in Pader District - } \\
\text { Northern Uganda: a } \\
\text { mixed methods } \\
\text { study }\end{array}$ & & & & & & $\begin{array}{l}\text { facility, } 50 \% \text { with a delay of } \\
\text { at least one month. } 12.9 \% \\
\text { sought help from } \\
\text { traditional healers and } \\
8.8 \% \text { self-medicated. }\end{array}$ & \\
\hline 73 & 2016 & $\begin{array}{l}\text { An exploration of } \\
\text { caregiver burden } \\
\text { for children with } \\
\text { nodding syndrome } \\
\text { (lucluc) in Northern } \\
\text { Uganda }\end{array}$ & Uganda & $\mathrm{N} / \mathrm{A}$ & Qualitative & $\begin{array}{c}54 \\
\text { caregivers }\end{array}$ & - & $\begin{array}{l}\text { Caregivers have a high } \\
\text { burden of care and } \\
\text { associated emotional } \\
\text { agony, anxiety for their } \\
\text { patient's safety and own } \\
\text { risk of burnout, face social } \\
\text { isolation and } \\
\text { stigmatisation and } \\
\text { homicidal and suicidal } \\
\text { ideations were recorded. }\end{array}$ & BMC Psychiatry \\
\hline 83 & 2017 & $\begin{array}{l}\text { Ivermectin } \\
\text { treatment in } \\
\text { patients With } \\
\text { onchocerciasis- } \\
\text { associated } \\
\text { epilepsy: protocol } \\
\text { of a randomized } \\
\text { clinical trial }\end{array}$ & $\begin{array}{l}\text { Democratic } \\
\text { Republic of } \\
\text { Congo }\end{array}$ & 2017-ongoing & $\begin{array}{l}\text { Randomised } \\
\text { controlled } \\
\text { trial protocol }\end{array}$ & N/A & N/A & $\begin{array}{l}\text { Protocol for a RCT in OV+ } \\
\text { epilepsy patients, } \\
\text { comparing immediate and } \\
\text { delayed (after } 4 \text { months) } \\
\text { ivermectin treatment. All } \\
\text { patients will receive AEDs. }\end{array}$ & $\begin{array}{l}\text { JMIR research } \\
\text { protocols }\end{array}$ \\
\hline 19 & 2018 & $\begin{array}{l}\text { Treatment and } \\
\text { rehabilitation } \\
\text { outcomes of } \\
\text { children affected } \\
\text { with nodding } \\
\text { syndrome in } \\
\text { Northern Uganda: a } \\
\text { descriptive case } \\
\text { series }\end{array}$ & Uganda & 2012 & Retrospective & 32 & - & $\begin{array}{l}13 \text { months of nutritional } \\
\text { and multivitamin } \\
\text { supplementation reduced } \\
\text { the incidences of severe or } \\
\text { moderate wasting ( } 9.7 \% \text { to } \\
2.6 \% \text { and } 19.7 \% \text { to } 2.6 \% \\
\text { respectively) and severe } \\
\text { and moderate stunting } \\
\text { (combined } 54.8 \% \text { to } 12.8 \% \\
\text { and } 7.7 \% \text { respectively). } \\
\text { AED therapy for } 29 \text { cases } \\
\text { was detailed, although } \\
\text { reduction in seizure } \\
\text { frequency not clearly } \\
\text { reported. Higher seizure } \\
\text { frequency associated with } \\
\text { female gender or febrile }\end{array}$ & $\begin{array}{l}\text { Pan African } \\
\text { Medical Journal }\end{array}$ \\
\hline
\end{tabular}


Aetiologies and therapies of nodding syndrome: a systematic review and meta-analysis

\begin{tabular}{|c|c|c|c|c|c|c|c|c|c|}
\hline Citation & $\begin{array}{l}\text { Publication } \\
\text { date }\end{array}$ & Title & Study location & Study date & Study design & Cases & Controls & Key finding(s) & Journal \\
\hline & & & & & & & & illness. & \\
\hline 82 & 2018 & $\begin{array}{l}\text { Setting up a clinical } \\
\text { trial for a novel } \\
\text { disease: a case } \\
\text { study of the } \\
\text { doxycycline for the } \\
\text { treatment of } \\
\text { nodding syndrome } \\
\text { trial - challenges, } \\
\text { enablers and } \\
\text { lessons learned }\end{array}$ & Uganda & 2015 & $\begin{array}{l}\text { Randomised } \\
\text { controlled } \\
\text { trial protocol }\end{array}$ & 115 & 115 & $\begin{array}{l}\text { NS patients will be given } 6 \\
\text { weeks of doxycycline } \\
\text { (100mg/day) or a placebo. } \\
\text { Primary outcome: } \% \text { of } \\
\text { patients with anti-LM-1 Ig } \\
\text { at } 24 \text { mo post-treatment. }\end{array}$ & $\begin{array}{l}\text { RCT protocol } \\
\text { (NCT02850913) }\end{array}$ \\
\hline
\end{tabular}

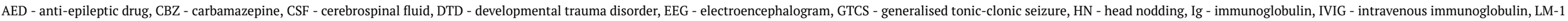
leiomodin-1, mo - month, Mt - mitochondrial, NS - nodding syndrome, OCE - other convulsive epilepsy, OV - Onchocerca volvulus, PHB - phenobarbitone, PHT - phenytoin, PTSD - post-traumatic stress disorder, RCT - randomised controlled tria 


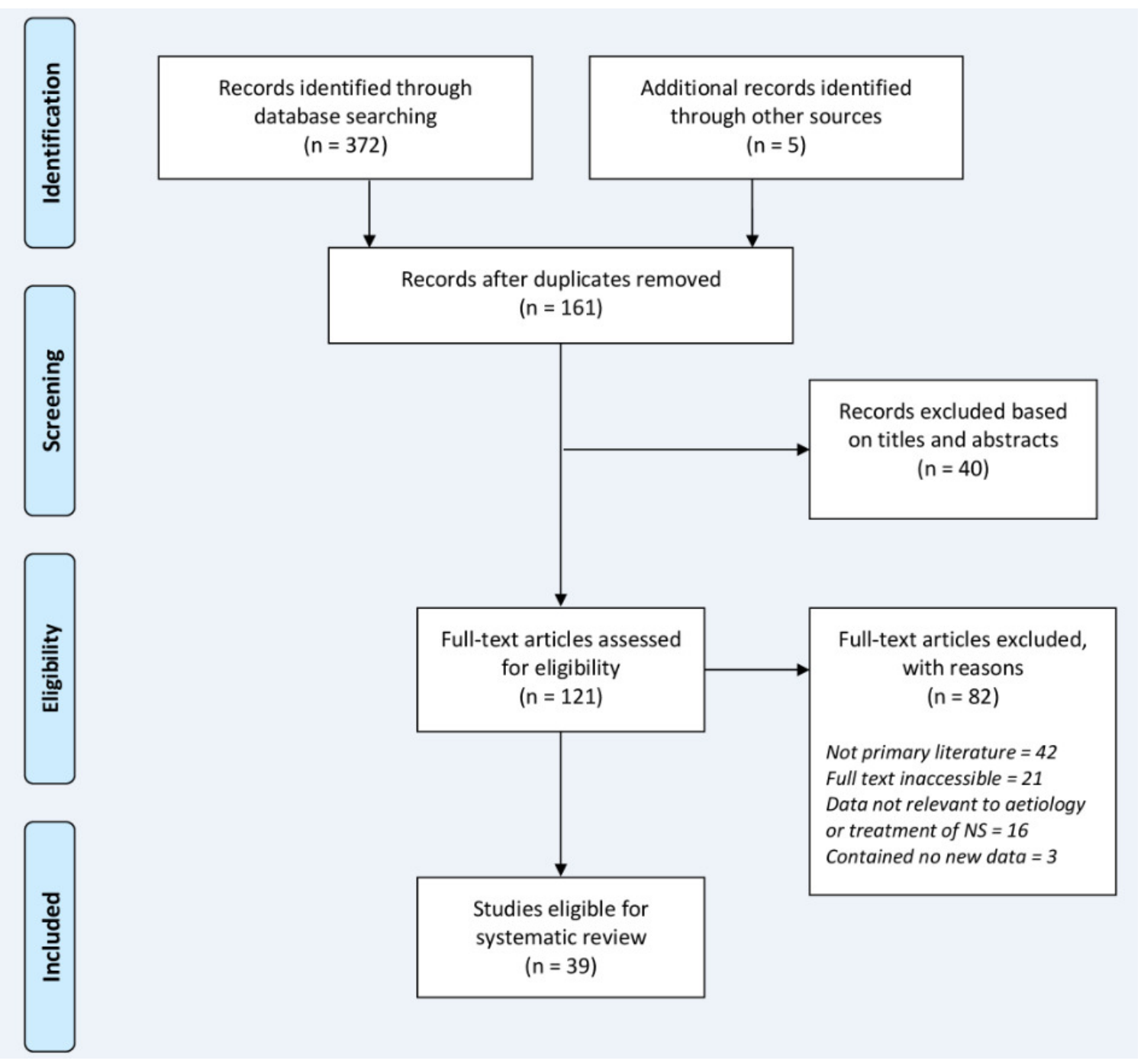

Figure 1. PRISMA flow diagram illustrating study selection.

PRISMA - Preferred Reporting Items for Systematic Reviews and Meta-Analyses

consumption of crushed cassava roots, but did not identify strong associations with the consumption of red sorghum or spoiled relief food. ${ }^{6}$ Notably, the consumption of certain cyanogenic plants, including sorghum and cassava, on the background of a protein-deficient diet has been linked to outbreaks of neurotoxic disease, albeit apparently not reminiscent of NS. ${ }^{40}$

It has also been postulated that mycotoxin-driven immunosuppression might have contributed to the NS outbreak, but mycotoxin levels have been shown to be similar between cases and controls ${ }^{28,39}$ and the distribution and clustering of cases does not appear to reflect an association with meals. ${ }^{46}$

Aside from the consumption of specific foodstuffs, the disruption of food security, food shortages and associated starvation have also been linked to the aetiology of NS. Several studies have investigated the presence of biomarkers for chronic malnutrition in NS patients. Vitamin B6 deficiency is known to cause abnormal tryptophan metabolism, leading to excessive production of 3-hydroxykynurenine which is known to be neurotoxic. ${ }^{47}$ Obol and colleagues studied 66 Ugandan cases and found that significantly lower vitamin B6 levels and high levels of 3-hydroxykynurenine were strongly associated with the presence of NS. ${ }^{36}$ In contrast, Foltz and colleague's earlier study of 42 Ugandan cases did not identify any significant association between NS and vitamin B6 deficiency. ${ }^{6}$ Moreover, a Tanzanian casecontrol study, which compared 22 NS patients to seven controls and one patient with generalised epilepsy, also failed to find an association between NS or epilepsy and vitamin B6 deficiency. ${ }^{27}$ A large anion gap, indicative of metabolic acidosis, has been observed in 112 NS patients across a case report, case series and case-control study. ${ }^{32-34}$ However, this likely reflects the patient's nutritional state, which could explain associations with a history of residence in IDP camps. ${ }^{34}$ It has also been suggested that this metabolic acidosis may reflect underlying mitochondrial dysfunction $^{32-34}$; however, analysis of three cases did not detect any aberrations in mitochondrial DNA. ${ }^{48}$ The serum biotinidase and acetyl carnitine levels of 47 Ugandan NS cases were found to be abnormally low, ${ }^{25}$ generating speculation that NS may represent the influence of metabolic disruption upon the central nervous system (CNS), ${ }^{16}$ as has been hypothesised for autism-spectrum disorders. ${ }^{49-51}$ Vitamin D 
deficiency has also been noted among NS patients, and could reflect more time spent indoors due to stigmatisation, the use of anticonvulsants or an aspect of the condition itself. 37

\section{PSYCHIATRIC}

Many children had horrific experiences during the conflicts that ravaged parts of Uganda and South Sudan in the 1990s and 2000s, while the URT remained comparatively stable. Psychiatric aetiologies for NS have therefore been considered and a sub-classification of 'NS psychiatric' (negative EEG and EMG findings), 'NS neurological' (positive electroencephalogram [EEG] and electromyograph [EMG] findings), 'NS mixed' and 'NS atypical' was proposed. ${ }^{52}$ However, detailed EEG analysis of two patients has demonstrated that head nodding episodes represent atonic, not 'pseudo', seizures. ${ }^{38}$ Musisi and colleagues found that many NS patients had complex psychiatric morbidities, some with a form of post-traumatic stress disorder known as developmental trauma disorder, and visual hallucinations were also reported. ${ }^{52}$ A 2013 case series of 22 patients by Idro and colleagues found that $12 / 22$ had psychiatric morbidities including hallucinations $(n=6)$, aggression $(n=5)$ and other behavioural problems $(n=4) .{ }^{29}$ An association between NS and visual hallucinations was also reported in a 2009 case-control study by Foltz and colleagues, but no association was found with the abduction of a family member or auditory hallucinations. ${ }^{6}$ In contrast, a recent case series involving 48 NS patients found no association between NS and visual hallucinations. ${ }^{25}$ Unpublished results from a 2016 cross-sectional study of 225 NS patients found that 'the majority' began head nodding in the IDP camps and also found that associated psychiatric comorbidities included depression, generalised anxiety disorder, post-traumatic stress disorder and pervasive developmental disorder. ${ }^{42}$ In a 2018 case series of 32 NS patients that had been resident in IDP camps, parents reported depression (50\% of cases), aggression (48.1\%), loss of appetite (39\%) and anxiety (14.3\%); $23.1 \%$ of these NS patients reported suicidal thoughts. ${ }^{19}$

It has been reported that for some patients episodes of head nodding may be spontaneous or triggered by cool temperatures, the offer of food, hyperventilation or sleep. ${ }^{29,38,53}$ Remarkably, head nodding has been reported to be triggered by the consumption of local, but not imported, foodstuffs. ${ }^{7}$ The psychological and neurological triggering of head nodding episodes requires greater investigation, and may contribute to the pathophysiological understanding.

\section{INFECTIOUS ASSOCIATIONS}

There has been a consistent epidemiological association in the literature between NS and the filarial nematode OV. 6,10,30,43,54 An association with another filarial nematode, Mansonella pestans (MP), has also been reported. ${ }^{7}$ There are two predominant theories regarding the role of filarial infection.

The first is that OV infection indirectly leads to NS via an autoimmune aetiology (see below). Cases of NS have thus far only been identified in areas endemic for OV. ${ }^{55}$ Temporal, familial and geographical clustering have been observed, ${ }^{56,57}$ for example limited mapping of NS cases has revealed clustering around watercourses, compared with other forms of epilepsy. ${ }^{30}$ Regional conflict has also caused disruption of OV elimination programmes, and may have resulted in increased prevalence. ${ }^{56}$ Moreover, regions in which community-directed treatment with ivermectin and larviciding of major watercourses have been reinstated have experienced a substantial drop in NS cases. ${ }^{58,59}$

Nine and six studies contained skin snip and/or polymerase chain reaction (PCR) data about the prevalence of OV among NS patients and non-epileptic controls respectively (Table 4). Among these studies there was limited, but conflicting, evidence about the association of ivermectin treatment with the prevalence of NS specifically. $7,33 \mathrm{We}$ found that four of the six studies that contained OV prevalence data about both NS patients and controls identified a statistically significant difference between both populations, however many of these studies used small sample sizes. To mitigate against this we performed random-effects meta-analyses to examine the prevalence of OV among NS cases and controls. Among a pooled population of $416 \mathrm{NS}$ patients we found that $68.0 \%(95 \% \mathrm{CI}=45.2-87.0)$ were $\mathrm{OV}+$ (Figure 2). Among a pooled population of 321 control subjects $46.6 \%$ (95\% CI=16.4-78.1) were OV+ (Figure 3). Both results were significant but there was a high level of heterogeneity among both cases $\left(\mathrm{I}^{2}=95.5 \%, 95 \% \mathrm{CI}=93.3-97.0\right)$ and controls ( $\mathrm{I}^{2}=97.1 \%, 95 \% \mathrm{CI}=95$ 95.5-98.2). The difference of $21.4 \%$ in OV prevalence between the pooled populations was significant $(P<0.0001)$.

Despite the strong association with the presence of microfilariae in the skin, and magnetic resonance imaging (MRI) evidence of gross neurological lesions in some NS patients, to date evidence of microfilariae in the cerebrospinal fluid (CSF) of NS patients has not been forthcoming. 4,6,7,38,40,43 A 2010 survey of CSF from 197 Tanzanians representing healthy, OV+ epileptic, OV+ only or epilepsy only patients did not yield evidence of OV in CSF, nor was skin snip density found to differ between groups. ${ }^{60}$ However, there is one report from 1976 of microfilariae in the CSF of $5 / 8$ heavily infected onchocerciasis patients without recorded NS or epilepsy. ${ }^{61}$

The second theory is that OV, and potentially MP, represent opportunistic infections in patients with immunosuppressive neurotropic viral infections, such as MV. ${ }^{41}$ Conflict-related disruption of immunisation programmes contributed to a large MV epidemic in northern Uganda between 1997 and 2003, with approximately 40 - 50,000 cases per year. There were also simultaneous increases, albeit of lower magnitude, in cases in South Sudan and the URT. It has been observed that the peak in this outbreak preceded the peak in NS cases by 5-6 years. ${ }^{41}$ This led to the hypothesis that NS may represent a post-infectious sequelae of MV, much like subacute sclerosing panencephalitis (SSPE), reported to occur in 4-27.4 cases per $100,000 .{ }^{62}$ Clinical similarities between NS and SSPE have been reported, and the two conditions have been compared elsewhere. ${ }^{41}$ However, NS has not been reported from other countries with past or present outbreaks of MV. 
Table 4. Studies with data about the prevalence of OV in NS cases and/or controls

\begin{tabular}{|c|c|c|c|c|c|c|c|c|c|c|c|c|c|c|}
\hline Citation & $\begin{array}{l}\text { Publication } \\
\text { date }\end{array}$ & Location & $\begin{array}{l}\text { Study } \\
\text { date }\end{array}$ & Cases & Controls & $\begin{array}{l}\text { OV+NS } \\
\text { patients }\end{array}$ & $\begin{array}{l}\text { Total NS } \\
\text { patients }\end{array}$ & $\%$ & $95 \% \mathrm{Cl}$ & $\begin{array}{c}\mathrm{OV}+ \\
\text { controls }\end{array}$ & $\begin{array}{c}\text { Total } \\
\text { control }\end{array}$ & $\%$ & $95 \% \mathrm{Cl}$ & $\mathrm{p}$ \\
\hline 43 & 2008 & Tanzania & $\mathrm{N} / \mathrm{A}$ & 62 & - & 43 & 51 & 84.3 & $71.4-93.0$ & - & - & - & & - \\
\hline 26 & 2012 & $\begin{array}{l}\text { South } \\
\text { Sudan }\end{array}$ & 2010 & 38 & 38 & 29 & 38 & 76.3 & $59.8-88.6$ & 18 & 38 & 47.4 & $31.0-64.2$ & 0.010 \\
\hline 7 & 2012 & $\begin{array}{l}\text { South } \\
\text { Sudan }\end{array}$ & 2001-02 & 82 & 81 & 76 & 82 & 92.7 & 84.8-97.3 & 39 & 81 & 48.1 & $36.9-59.5$ & $<0.0001$ \\
\hline 6 & 2013 & Uganda & 2009 & 49 & 49 & 32 & 45 & 71.1 & $55.7-83.6$ & 21 & 39 & 53.8 & $37.2-69.9$ & 0.1034 \\
\hline 29 & 2013 & Uganda & 2012 & 22 & - & 3 & 10 & 30.0 & $6.7-65.2$ & - & - & - & & - \\
\hline 33 & 2013 & Uganda & 2012 & 101 & 101 & 16 & 101 & 15.8 & $9.3-24.4$ & 0 & 101 & $0.0^{*}$ & $0.0-3.6$ & $<0.0001$ \\
\hline 44 & 2013 & Tanzania & N/A & 12 & 20 & 9 & 12 & 75.0 & $42.8-94.5$ & - & - & - & & - \\
\hline 27 & 2014 & Tanzania & $N / A$ & 22 & 8 & 17 & 22 & 77.3 & $54.6-92.1$ & 7 & 7 & $100^{*}$ & $59.0-100.0$ & 0.1736 \\
\hline 8 & 2017 & N/A & N/A & 55 & 55 & 44 & 55 & 80.0 & $67.0-89.6$ & 29 & 55 & 52.7 & $38.8-66.3$ & 0.0026 \\
\hline
\end{tabular}

*Assumed 0.01 or 99.9 to permit calculation of significance. CI -confidence intervals, NS - nodding syndrome, OV - Onchocerca volvulus 


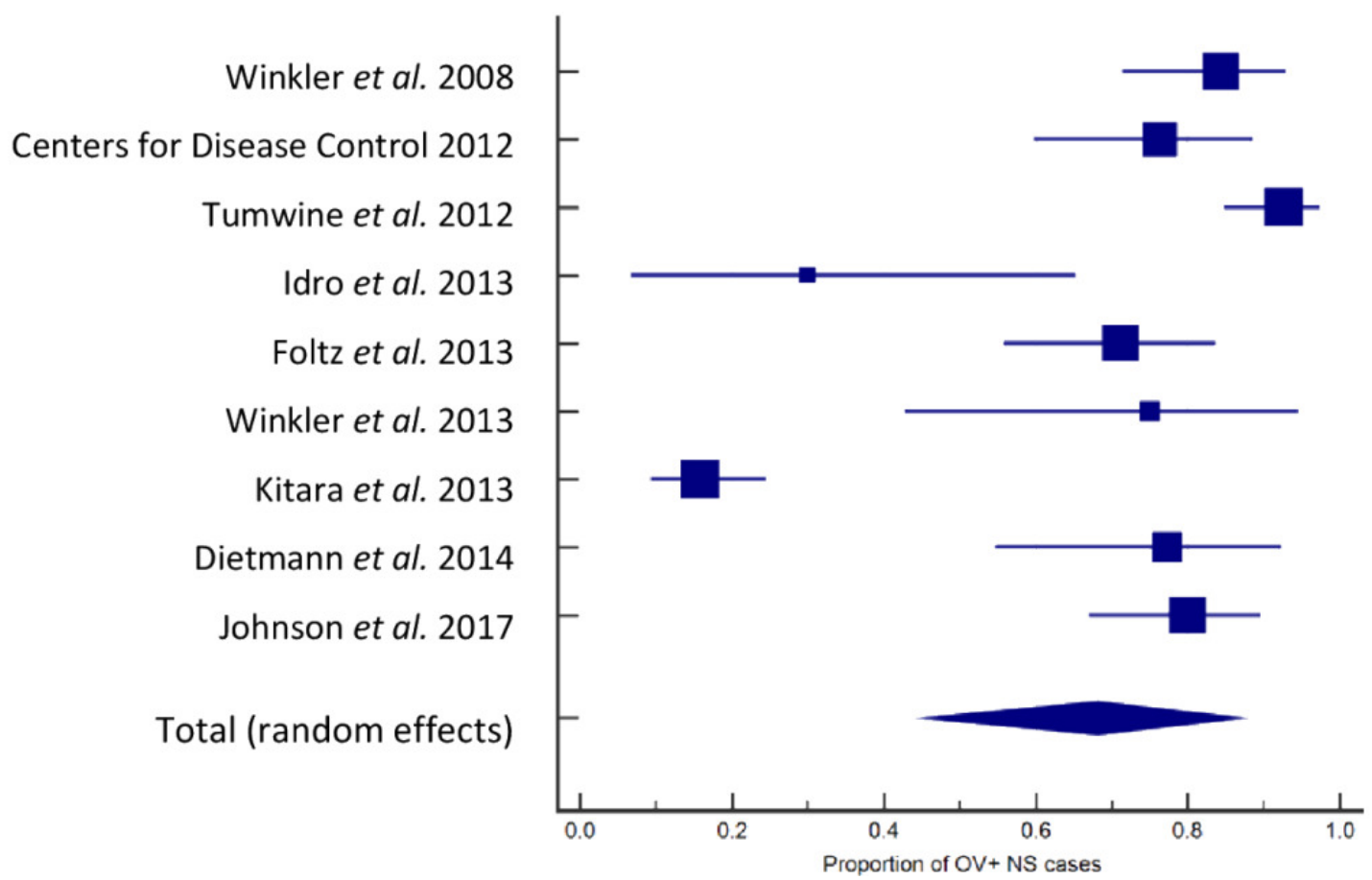

Figure 2. Forest plot of the prevalence of $\mathrm{OV}$ in NS patients $(n=416)$.

Blue boxes show effect estimates for each study; horizontal lines indicate $95 \%$ CIs. The diamond shows the pooled prevalence of OV, with the centre being the pooled estimate and tips 95\% confidence intervals; NS, nodding syndrome; OV, Onchocerca volvulus.

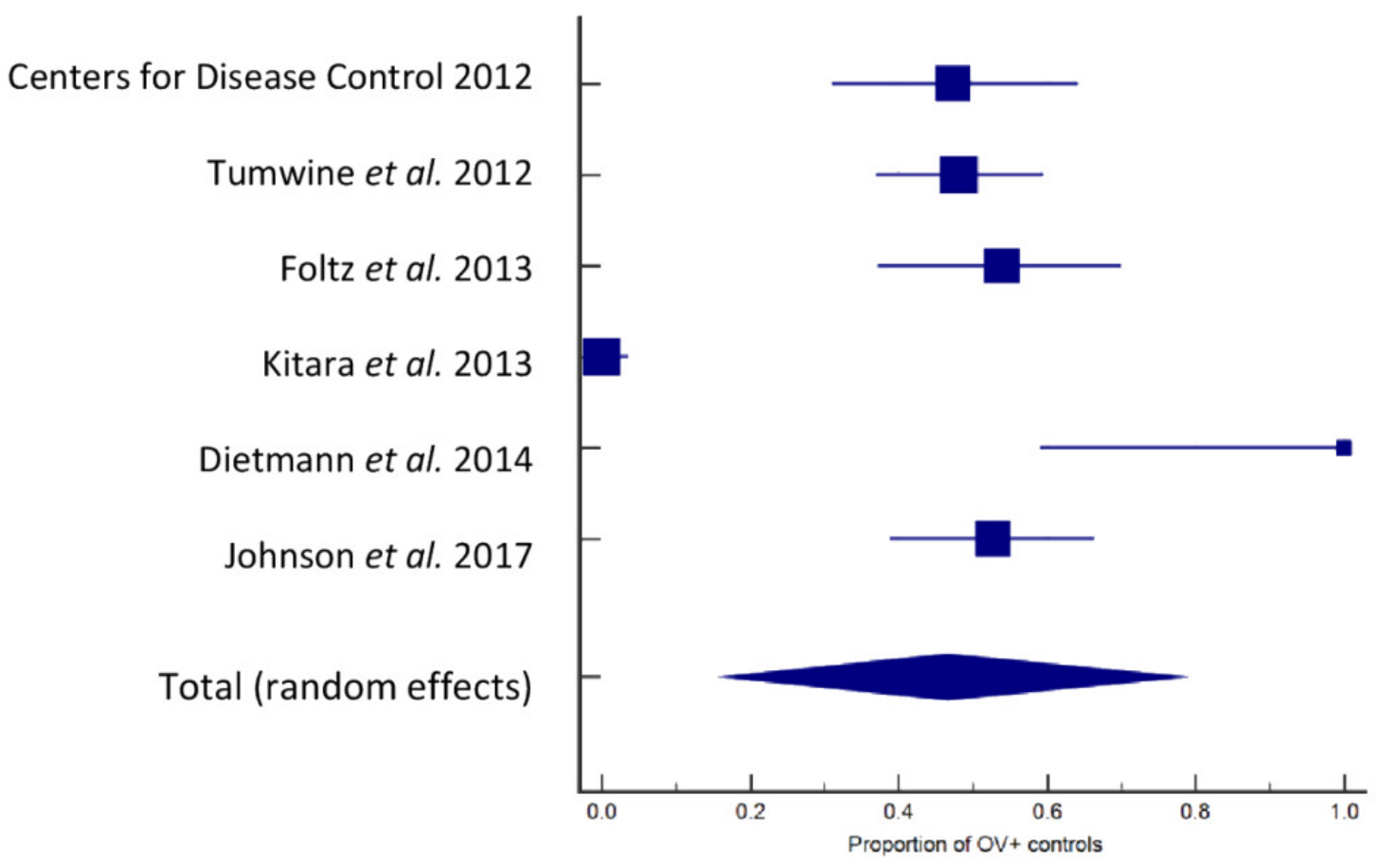

Figure 3. Forest plot of the prevalence of OV in control subjects $(n=321)$.

Blue boxes show effect estimates for each study; horizontal lines indicate $95 \%$ CIs. The diamond shows the pooled prevalence of OV, with the centre being the pooled estimate and tips $95 \%$ confidence intervals; NS, nodding syndrome; OV, Onchocerca volvulus.

Three case-control studies have investigated the association between MV and NS; two studies from northern Uganda and one from South Sudan.6,7,41 Together these three studies examined 112 cases and 118 controls for the association of a caregiver's report of previous MV infection and NS. Spencer and colleagues reported a seemingly strong association between previous MV infection and NS, although the $95 \%$ CIs were wide (odds ratio $(\mathrm{OR})=6.00,95 \%$ 
$\left.\mathrm{CI}=1.03-113^{22}\right)$. In contrast, Foltz and colleagues found a positive, albeit weaker, association $(\mathrm{OR}=4.0,95 \%$ $\mathrm{CI}=1.1-14.2$; adjusted odds ratio $(\mathrm{AOR})=3.3, \quad 95 \%$ $\left.\mathrm{CI}=0.8-13.6^{6}\right)$. In a smaller study from South Sudan, Tumwine and colleagues examined 13 cases and 19 controls and found no evidence of an association ( $\mathrm{OR}=0.13$, 95\% $\left.\mathrm{CI}=0.02-0.76^{7}\right)$. A medical history of MV infection is however open to recall bias, Foltz and colleagues used PCR to investigate the presence of MV in the CSF of 16 NS cases, all of which were negative. 6

Immunosuppression, linked to mycotoxin exposure and malnutrition, has been suggested to have contributed to the rise in MV cases, and to partly explain the higher prevalence of NS among the Moru compared with the Dinka peoples in South Sudan 7,41,63; this disparity has since been disputed. ${ }^{46}$ Alternatively, it has been hypothesised that different farming practices, the Moru being more reliant on crops and the Dinka on livestock, may have allowed the Dinka to acquire cross-immunity between MV and rinderpest, a related morbillivirus of ruminants. ${ }^{41}$ Rinderpest has been absent from Africa since 2001, and it has been suggested that loss of cross-immunity could explain the occurrence of NS among the Dinka after that date. ${ }^{41,42}$

While the neuropathology of NS remains to be fully elucidated, crystalline inclusions have been observed in histological sections from 6/9 patients. ${ }^{30}$ Given the absence of MRI evidence for crystalline material, it has been suggested that these may represent post-mortem artefacts. ${ }^{64}$ Characterisation of these postulated inclusion bodies is required, given its reported similarity to NS it is intriguing that the histopathology of SSPE is characterised by the presence of distinct inclusion bodies. ${ }^{65}$

\section{AUTOIMMUNITY}

Autoantibodies have been strongly associated with some forms of epilepsy and other neurological disorders. ${ }^{66}$ Like an infectious aetiology, autoimmunity offers a potential explanation for a range of spatially distributed CNS lesions. Although gross CNS pathology has been observed using imaging studies in some NS patients, evidence of neuroinflammation associated with an autoimmune response has to the best of our knowledge not yet been published. $4,29,38,44$ Nevertheless, there have been investigations into the presence of autoantibodies in NS patients. Idro and colleagues identified autoantibodies against the voltage-gated potassium channel (VGKC) in 15/31 NS patients compared to $1 / 11$ controls, but there was no evidence of antibodies against the intracellular glutamic acid decarboxylase, associated with complex epilepsy. ${ }^{30}$ These data contrasted with that of Dietmann and colleagues who failed to identify VGKC or N-methy-D-aspartate receptor autoantibodies ${ }^{27}$; although the sample storage and assays used in this study have been questioned. ${ }^{30}$ Meanwhile, Soldatos and colleagues examined the CSF of two of three NS patients and found evidence of partially identical oligoclonal immunoglobulin bands. ${ }^{48}$

In 2014 it was suggested that autoantibodies against the autoantigen Ieiomodin-1 (anti-LM-1 immunoglobulin [Ig]) may be associated with NS, with anti-LM-1 Ig present in 11/19 Ugandan cases compared to 5/19 local matched con- trols. ${ }^{31} \mathrm{LM}-1$ has been reported to be expressed intra- and extra- cellularly in neurons, and is known to be an important component of the actin-myosin complex in myocytes. ${ }^{67,68}$ The expression of LM-1 in the murine CNS closely matches regions observed to be affected in NS and anti-LM-1 Ig has been shown to be neurotoxic to murine CNS tissue. ${ }^{8}$ Moreover, anti-LM-1 Ig exhibits cross-reactivity with OV tropomyosin, with which LM-1 shares $25.5 \%$ primary sequence identity (37\% similarity) and has localised regions of higher sequence homology, e.g. LKEAExRAE is $66.7 \%$ identical to amino acids 362 and 372 of LM-1. ${ }^{8}$ Such cross-reactivity is suggestive of autoimmunity driven by molecular mimicry.

This initial report was followed by a study that investigated the presence of anti-LM-1 Ig in the serum of a further 36 patients from South Sudan. The infection status of the patients was determined by serology (cohort one) or skin snip (cohort two). 8,31 Together, anti-LM-1 Ig was present in the serum of $29 / 55$ (52.7\%) of the pooled population of NS patients compared with $17 / 55$ unaffected pooled controls (30.8\%; $P=0.024$; matched OR=2.7 (1.1-6.5)). Notably, 24/44 (54.5\%) OV+ NS patients were also positive for anti-LM-1 Ig, while 12/29 (41.3\%) OV+ controls were also positive for anti-LM-1 lg. The difference in the presence of serum antiLM-1 Ig between the NS and control cohorts (including OV positive $(+)$ and OV negative (-) patients) was reported as significant $(P=0.024)$. However, we compared the presence of anti-LM-1 Ig between OV+ patients or OV+ controls and found a $13.2 \%$ difference that was not significant $(P=0.273$; 95\% CI=-9.81-34.2). CSF samples taken from 16 NS patients were also examined and 50\% contained anti-LM-1 Ig, compared to 0/8 North American epilepsy patients. ${ }^{8}$

\section{THERAPEUTIC APPROACHES TO NS}

NS shows an almost invariably progressive course, although treatment can improve life expectancy. ${ }^{15}$ The condition carries a high burden of social stigma for both patients and their families, ${ }^{69,70}$ including negative stereotypes from healthcare workers. ${ }^{71}$ Some NS patients have been isolated by communities afraid of contagion. ${ }^{53}$ Given the typical age and impaired abilities of NS patients, caregivers are vital in facilitating treatment; they seek help from a variety of sources including health facilities (78\%), traditional healers (13\%) and self-medication (9\%). Half of those who attended health facilities did so a month after the onset of symptoms, indicating that more work is needed to ensure prompt help is sought. ${ }^{72}$ Aside from the work on social stigma there has been little work on the impact of NS on caregivers, although one qualitative study identified a high burden of emotional agony, alongside issues surrounding burnout, safety concerns, stigmatisation and rejection and homicidal ideation. ${ }^{73}$ Psychological interventions have been shown to mitigate depression in caregivers. ${ }^{74}$

The 2013 guidelines for the management of NS suggest that, depending on severity, care may include anti-epileptics or anti-convulsants, appropriate management of comorbidities, psychiatric support, nutritional assistance and physical, occupational, speech and language therapy. ${ }^{5} \mathrm{~A}$ cross-sectional cohort study of NS and other convulsive epilepsy (OCE) patients treated according to these guide- 
lines showed improvement in both groups of patients, with $25 \%$ of NS and $51 \%$ of OCE patients being seizure free after 12 months. ${ }^{15}$

The 2013 guidelines detail the usage, advantages and disadvantages of anti-epileptics for NS. ${ }^{5}$ However, despite successful reports of the use of anti-epileptics, no clinical trial has been performed. Successful use of sodium valproate has been reported, ${ }^{75}$ and a case-control study showed a $57 \%$ reduction in total seizure burden among 22 NS patients treated with sodium valproate for $2-3$ weeks. ${ }^{5}$ A 1994 case report demonstrated some initial efficacy for phenobarbitone, but long-term control was not achieved. ${ }^{11}$ A 2013 case series in which four patients were treated with anti-epileptics provided mixed or no evidence for the efficacy of carbamazepine and phenobarbitone respectively. ${ }^{52}$ In 2014 Winkler and colleagues demonstrated cessation or reduction of head nodding frequency in the majority of patients treated with anti-epileptic drugs. ${ }^{76}$ However, in 2015 de Polo and colleagues treated 21 patients with carbamazepine as a mono- or combination therapy and found that none obtained good control of any form of seizure associated with NS. ${ }^{77}$ Benzodiazepines are indicated for icteric patients, ${ }^{5}$ and given the symptomatic overlap between NS and catatonia, lorazepam has been piloted in NS patients. ${ }^{78}$ Within one hour of lorazepam administration the severity of catatonia symptoms was reduced in 10/16 patients, and by more than $50 \%$ in six. ${ }^{78}$

Mental health comorbidities have been observed among NS patients, including post-traumatic stress disorder and depression. ${ }^{52}$ Use of the tricyclic antidepressant imipramine has been recorded for three cases, in which the mood of two improved. However, it was administered as part of a wider treatment programme and the precise contribution of imipramine was unclear. ${ }^{52}$

Finally, there are treatments aimed at potential aetiologies. Antibiotic elimination of the symbiotic Wolbachia bacteria from the OV nematode has been shown to reduce the inflammation caused by, and contribute to premature death of, the nematode. ${ }^{79,80}$ An ongoing clinical trial is investigating doxycycline for the treatment of NS, with the aim of elucidating whether Onchocerca or Wolbachia may drive the disease. ${ }^{80,81}$ There is also an ongoing clinical trial in the Democratic Republic of Congo examining the effect of ivermectin administration in reducing the frequency of $\mathrm{OAE}$, which may have implications for NS patients. ${ }^{82}$ Three OVNS patients have been treated with plasmapheresis or intravenous immunoglobulin for presumed autoimmunity, however the results have not been recorded in sufficient detail to appraise treatment efficacy. ${ }^{48}$

\section{DISCUSSION}

Although it is 53 years since NS was first described, the underlying aetiology and pathogenesis remain unclear. This systematic review has collated evidence for metabolic, psychogenic, infectious and autoimmune theories, the limitations of which will now be discussed. Whatever the aetiology, it must explain the age range of patients and disparate nature of clinical findings. ${ }^{29,38,43,44}$ Notably, probable cases dating from 1934 would call into question hypotheses based solely on modern phenomena. ${ }^{2}$
The epidemiological history of NS is intriguing: an endemic pattern of cases in the URT and one large epidemic among a broadly malnourished and traumatised Ugandan and South Sudanese population that appears to have a 5-7 year temporal disparity with conflict, food shortages and/or MV epidemics, and no new epidemic cases since 2013. Familial clustering has also been observed, with one case series reporting that $79.6 \%$ of NS patients had a family history positive for NS or epilepsy. ${ }^{17,76}$ Given this apparent familial clustering of NS cases within blackfly exposed locales, genetic and epigenetic genome-wide association studies (GWAS) are warranted and could offer insight into the aetiology of NS and differential susceptibility. The epidemic versus endemic nature of NS is important and calls into question metabolic or psychiatric associations with relief food, IDP camps, conflict and psychological trauma. ${ }^{32,83} \mathrm{Al}-$ ternatively, NS in the URT may represent a distinct condition. ${ }^{83}$ It has been suggested that if NS is driven by a specific pathogen, an epidemic could reflect the movement of a non-immune population into an affected area. ${ }^{46}$ However, if an OV-driven autoimmune aetiology is ultimately described, then regional differences between OV, Wolbachia or the blackfly could explain the differential presence or manifestation of NS. Aside from infectious or immunological theories, observed correlations with biomarkers for malnutrition remain unexplained, although these may be incidental.

The epidemiological association between OV and NS has been questioned. ${ }^{84}$ OV occurs in 34 countries in Africa, the Middle East, South and Central America, with an estimated 17.7 million people affected. ${ }^{85}$ In contrast, there have only been several thousand cases of NS in restricted parts of Africa. ${ }^{3}$ An attempt to resolve this discrepancy has been made by the recent classification of NS under the umbrella term OAE. ${ }^{9}$ There is substantial evidence in favour of $\mathrm{OAE},{ }^{10}$ and NS is presumed to represent a point on this proposed clinical spectrum, much of which may remain unappreciated. Meta-analysis of the relationship between OV and epilepsy demonstrated a pooled OR of 2.49 (95\% $\mathrm{CI}=1.61-3.86 ; \quad P<0.001)$, or 1.29 (95\% CI=0.93-1.79; $P=0.139$ ) when controlled for age, residence and gender 86 ; a significant association was found using studies that reported the presence of OV nodules. ${ }^{86}$ A recent prospective study from an OV focus in Cameroon has demonstrated a relationship between childhood skin microfilarial density and subsequent risk of epilepsy or seizures. ${ }^{12}$ Moreover, a fall in the number of cases of NS, as well as cases of epilepsy and Nakalanaga syndrome, appears to correlate with the reestablishment of onchocerciasis control. ${ }^{59}$ Such correlations could be investigated by further prospective studies. Nevertheless, it is unclear, given the large numbers of OV patients, why NS has not been documented in other OV-endemic regions, although this could reflect greater OV elimination efforts or genotypic differences between populations. ${ }^{51,87,88}$ Moreover, the interpretation of case-control studies, including those included in the meta-analysis, may be limited by the availability of data about ivermectin therapy given to individuals in such studies. If NS is indeed associated with OV then administration of ivermectin to NS patients may confound observations of this association. It would be ideal if all case-control studies clearly reported 
ivermectin administration data pertaining to periods before and after the onset of NS, however many studies do not.

The clinical similarities between NS and SSPE have been discussed elsewhere, ${ }^{41}$ however the association with MV remains unclear. The only significant OR reported had very wide confidence intervals, ${ }^{41}$ suggesting the study should be repeated with a larger sample size. There is limited correlative evidence suggesting an association between MV and NS epidemics, ${ }^{41}$ the authors are not aware of reports of NS-like disease in other MV endemic areas, and seizures associated with some cases of MV infection may have led to some patients incorrectly being classified as NS cases. ${ }^{45}$ Although MV is immunosuppressive, ${ }^{89}$ it is noted that for immunosuppressed patients to only acquire OV or MP as opportunistic infections is unusual. In contrast, acquired immunodeficiency syndrome patients readily acquire a range of opportunistic infections, including common fungal infections of which there is no recorded association with NS. 90 Moreover, it has been suggested that the crystalline inclusions thought to mimic those observed in SSPE represent post-mortem artefacts. ${ }^{64}$ Histopathological investigations are ongoing, and recent unpublished investigations suggest that NS may be a unique neurodegenerative disorder comparable to a frontotemporal degenerative tauopathy. ${ }^{91}$

An autoimmune reaction driven by molecular mimicry between OV and human LM-1 was recently proposed. ${ }^{8}$ However, while Johnson and colleagues demonstrated an association between the presence of anti-LM-1 Ig (subtypes $\mathrm{G}$ and $\mathrm{M}$ ) and NS, whether anti-LM-1 Ig contributes to, is a consequence of or is coincidental with the pathology of NS remains unclear. The naturally occurring presence of autoreactive B cells is well documented. ${ }^{92}$ While autoantibodies can cause pathology, they can also arise because of cell damage, which may occur secondary to seizures. ${ }^{63}$ Autoimmune conditions are often associated with inflammatory responses and, at the time of writing, the current lack of evidence for neuroinflammation in NS patients remains intriguing. Moreover, despite expression of LM-1 in myocytes, there are no reports of autoimmune myopathy among NS patients. ${ }^{63}$ The antibody subtypes identified were IgG and IgM. ${ }^{8}$ The latter could suggest an ongoing immune response, and subtyping of IgG could offer further insights. The response of NS patients to carefully managed corticosteroids could reveal whether sources of inflammation remain to be identified. The outcome of the treatment of three NS patients with IVIG or plasmapheresis has not yet been made available in detail, but will contribute to the debate. ${ }^{48}$ The molecular mimicry theory must also explain the presence of NS in OV- patients, although it's unclear whether or not these were OV naïve patients. ${ }^{8}$ The presence of OV+ NS patients negative for anti-LM-1 Ig could be explained by detection difficulties or the gradual loss of antibodies following an initiating event, however many autoimmune conditions are chronic and progressive.

Limitations of this review include the exclusion of a small number of studies that were not available in full-text form (Appendix S3, Online Supplementary Document). For their studies to have the greatest impact in the field authors should strive to publish in open-access indexed journals. Moreover, publication of the same data multiple times, encountered several times, does not increase its impact.
Assessment of the titles, and where possible abstracts, of these studies suggested that their omission is unlikely to have greatly altered this review. Five potentially relevant studies were not identified by the systematic search strategy. These may have been incorrectly indexed and were included manually to ensure as much relevant data as possible was screened. This approach enhances the transparency and reproducibility of this study, although we cannot exclude the possibility that other non-indexed studies were unknowingly omitted. This study may also be limited by not being pre-registered. Registration of reviews is a nonessential recommendation designed to encourage transparency, improve quality and reduce duplication. Pre-registration of reviews that are never completed is not recommended. ${ }^{93}$ This study was conceived as a student project, many of which are not published, and the authors therefore decided it was inappropriate to register retrospectively. However, the authors are not aware of any similar studies underway and complied with the PRISMA statement throughout.

A further potential limitation of this study is its focus specifically on NS. While we appreciate that NS may be found to exist as part of a spectrum of OAE, the focus of this study was to review literature pertaining only to NS. A systematic review specifically exploring the link between epilepsy and OV was performed in $2013,{ }^{86}$ but as there has been much new work in this area an updated review could be considered. Lastly, it must be remembered that systematic reviews are only ever a snapshot of a dynamic literature and at the time of writing the NS field awaits clear and open reporting of important results, including ongoing trials, post-mortem analyses and immunomodulatory treatment efficacy. $38,61,81,94$

The aetiology of NS remains unclear with each of the proposed theories still having questions to address. Ongoing trials and further correlative work (e.g. GWAS) may help direct the field towards a conclusive mechanism. It is important to understand the aetiology of NS to care for those affected and to better prevent or treat future epidemics. It is intriguing that no new epidemic cases have been reported since 2013, but this will offer little reassurance until the aetiology and therapy of NS is better understood.

\section{ACKNOWLEDGEMENTS}

The authors wish to thank Dr Medhat Khattar (Division of Infection and Pathway Medicine, University of Edinburgh) for constructive criticism of the manuscript. We wish to thank the two anonymous reviewers of this manuscript for their constructive input.

\section{ETHICS APPROVAL AND CONSENT TO PARTICIPATE}

Not applicable.

\section{COMPETING INTERESTS}

The authors completed the Unified Competing Interest form at http://www.icmje.org/coi_disclosure.pdf, and declare no conflicts of interest. 


\section{FUNDING}

None. Access to MedCalc was through a license provided by the Biomedical Teaching Organisation, University of Edinburgh.

\section{CORRESPONDENCE TO:}

Dr Joshua D. Jones MA MRes PhD (Cantab)

Division of Infection Medicine
Edinburgh Medical School: Biomedical Sciences University of Edinburgh

1 George Square

Edinburgh EH8 9JZ

UK

josh.jones@ed.ac.uk 


\section{REFERENCES}

1. Aall-Jilek LM. Epilepsy in the Wapogoro tribe in Tanganyika. Acta Psychiatr Scand. 1965;41:57-58. do i:10.1111/j.1600-0447.1965.tb04970.x

2. Spencer PS, Palmer VS, Jilek-Aall L. Nodding syndrome: origins and natural history of a longstanding epileptic disorder in sub-Saharan Africa. Afr Health Sci. 2013;13(2):176-182. doi:10.431 4/ahs.v13i2.1

3. World Health Organisation. Onchocerciasis | Nodding syndrome. Accessed May 29, 2018. http://ww w.who.int/onchocerciasis/symptoms/nodding_syndro me/en/

4. Dowell SF, Sejvar JJ, Riek L, et al. Nodding Syndrome. Emerg Infect Dis. 2013;19(9):1374-1384. do i:10.3201/eid1909.130401

5. Idro R, Musubire KA, Byamah MB, et al. Proposed guidelines for the management of nodding syndrome. Afr Health Sci. 2013;13(2):219-232. doi:10.4314/ahs.v $\underline{13 i 2.4}$

6. Foltz JL, Makumbi I, Sejvar JJ, et al. An epidemiologic investigation of potential risk factors for nodding syndrome in Kitgum District, Uganda. PLoS One. 2013;8(6):e66419. doi:10.1371/journal.pon e.0066419

7. Tumwine JK, Vandemaele K, Chungong S, Richer M, Anker M, Ayana Y. Clinical and epidemiologic characteristics of nodding syndrome in Mundri County, southern Sudan. Afr Health Sci. 2012;12:242-248.

8. Johnson TP, Tyagi R, Lee PR, et al. Nodding syndrome may be an autoimmune reaction to the parasitic worm Onchocerca volvulus. Sci Transl Med. 2017;9(377):eaaf6953. doi:10.1126/scitranslmed.aaf6 953

9. Colebunders R, Njamnshi AK, van Oijen M, et al. Onchocerciasis-associated epilepsy: From recent epidemiological and clinical findings to policy implications. Epilepsia Open. 2017;2(2):145-152. doi:1 $\underline{0.1002 / \text { epi4.12054 }}$

10. Colebunders R, Mandro M, Njamnshi AK, et al. Report of the first international workshop on onchocerciasis-associated epilepsy. Infect Dis Poverty. 2018;7(1):23. doi:10.1186/s40249-018-0400-0

11. Kaiser C, Kipp W, Tukesiga E, Asaba G, Rubaale T. Nodding syndrome, western Uganda, 1994. Am J Trop Med Hyg. 2015;93:198-202. doi:10.4269/ajtmh.14-083 $\underline{8}$
12. Chesnais CB, Nana-Djeunga HC, Njamnshi AK, et al. The temporal relationship between onchocerciasis and epilepsy: a population-based cohort study. Lancet Infect Dis. 2018;18(11):1278-1286. doi:10.1016/s147 3-3099(18)30425-0

13. Casis Sacre G. El sindrome epileptico y sus reaciones con onchocercosis. Boletin Salubr E Hig. 1938;1:11-31.

14. Colebunders R, Hendy A, Nanyunja M, Wamala JF, van Oijen M. Nodding syndrome-a new hypothesis and new direction for research. Int I Infect Dis. 2014;27:74-77. doi:10.1016/i.ijid.2014.08.001

15. Idro R, Namusoke H, Abbo C, et al. Patients with nodding syndrome in Uganda improve with symptomatic treatment: a cross-sectional study. BMJ Open. 2014;4(11):e006476. doi:10.1136/bmjopen-201 4-006476

16. Arony DA, Gazda S, Kitara DL. Could nodding syndrome in Northern Uganda be a form of autism spectrum disorder? An observational study design. Pan Afr Med J. 2018;30:115. doi:10.11604/pamj.2018.3 0.115 .13634

17. Arony AD, Collines A, Frederick ME, Gazda S, Kitara DL. Is there a line between internal displacement; environmental and dietary factors in the onset of nodding syndrome in northern Uganda? A clinical observational study design. WIMPR. 2017;3:34-48.

18. Arony DA, Galloway P, Collines A, Frederick ME, Kitara DL. Metabolic analyses of nodding syndrome in Uganda: a pilot study is a biotinidase and acetyl carnitine deficiency; a metabolic disorder. An observational study design. World J Phamaceutical Med Res. 2018;4:160-174.

19. Gazda S, Kitara DL. Treatment and rehabilitation outcomes of children affected with nodding syndrome in Northern Uganda: a descriptive case series. Pan Afr Med J. 2018;29:228. doi:10.11604/pamj.2018.29.228.1 $\underline{3627}$

20. Winkler AS, Friedrich K, Meindl M, et al. Clinical characteristics of people with head nodding in southern Tanzania. Trop Doct. 2010;40(3):173-175. do $\underline{\mathrm{i}: 10.1258 / \mathrm{td} .2010 .090373}$

21. Moher D, Liberati A, Tetzlaff J, Altman DG, The PRISMA Group. Preferred Reporting Items for Systematic Reviews and Meta-Analyses: The PRISMA Statement. PLoS Med. 2009;6(7):e1000097. doi:10.137 1/journal.pmed.1000097 
22. The Joanna Briggs Institute. Critical appraisal tools - JBI. Accessed August 17, 2018. http://joannabri ggs.org/research/critical-appraisal-tools.html

23. Higgins JPT, Thompson SG, Deeks JJ, Altman DG. Measuring inconsistency in meta-analyses. BMJ. 2003;327(7414):557-560. doi:10.1136/bmj.327.7414.5 $\underline{57}$

24. Freeman MF, Tukey JW. Transformations related to the angular and the square root. Ann Math Stat. 1950;21(4):607-611. doi:10.1214/aoms/1177729756

25. Atim-Oluk MA, Galloway PG, Lagoro DKL. Metabolic analysis of children affected with nodding syndrome in north Uganda: A pilot study. Dev Med Child Neurol. 2017;59:60-61.

26. Centers for Disease C. Prevention. Nodding syndrome - South Sudan, 2011. MMWR Morb Mortal Wkly Rep. 2012;61:52-54.

27. Dietmann A, Wallner B, König R, et al. Nodding syndrome in Tanzania may not be associated with circulating anti-NMDA- and anti-VGKC receptor antibodies or decreased pyridoxal phosphate serum levels-a pilot study. Afr H Sci. 2014;14(2):434-438. do $\mathrm{i}: 10.4314 / \mathrm{ahs} . v 14 \mathrm{i} 2.20$

28. Duringer JM, Craig AM, Mazumder R, Palmer VS, Lasarev MR, Spencer PS. Nodding Syndrome: Multimycotoxin Case-Control Study in Northern Uganda. Ann Neurol. 2017;82:S171-2.

29. Idro R, Opoka RO, Aanyu HT, et al. Nodding syndrome in Ugandan children-clinical features, brain imaging and complications: a case series. $B M J$ Open. 2013;3(5):11. doi:10.1136/bmjopen-2012-0025 $\underline{40}$

30. Idro R, Opar B, Wamala J, et al. Is nodding syndrome an Onchocerca volvulus-induced neuroinflammatory disorder? Uganda's story of research in understanding the disease. Int I Infect Dis. 2016;45:112-117. doi:10.1016/j.ijid.2016.03.002

31. Johnson T, Tyagi R, Lee PR, et al. Detection of auto-antibodies to leiomodin- 1 in patients with nodding syndrome. J Neuroimmunol. 2014;275(1-2):103. doi:10.1016/j.jneuroim.2014.08.27 $\underline{5}$

32. Kitara D, Gazda S, Ambrose E, et al. Nodding Episodes and High Anion Gap in a 13 Year Old Child with Nodding Syndrome: A Case Report. Br J Med Med Res. 2015;6(8):851-858. doi:10.9734/bjmmr/2015/145 $\underline{95}$
33. Kitara DL, Mwaka AD, Anywar DA, Uwonda G, Abwang B, Kigonya E. Nodding Syndrome (NS) in Northern Uganda: a probable metabolic disorder. $\mathrm{Br} J$ Med Med Res. 2013;3(4):2054-2068. doi:10.9734/bjm $\mathrm{mr} / 2013 / 4357$

34. Kitara DL, Mwaka AD, Kigonya E. High anion gap metabolic acidosis among children with Nodding Syndrome (NS) in Northern Uganda: case series. $\mathrm{Br} \mathrm{J}$ Med Med Res. 2014;4(6):1301-1314. doi:10.9734/bjm $\underline{\mathrm{mr} / 2014 / 6151}$

35. Landis JL, Palmer VS, Spencer PS. Nodding syndrome in Kitgum District, Uganda: association with conflict and internal displacement. BMJ Open. 2014;4(11):e006195. doi:10.1136/bmjopen-2014-0061 $\underline{95}$

36. Obol JH, Arony DA, Wanyama R, et al. Reduced plasma concentrations of vitamin B6 and increased plasma concentrations of the neurotoxin 3-hydroxykynurenine are associated with nodding syndrome: a case control study in Gulu and Amuru districts, Northern Uganda. Pan Afr Med J. 2016;24:123. doi:10.11604/pamj.2016.24.123.8409

37. Piloya-Were T, Odongkara-Mpora B, Namusoke H, Idro R. Physical growth, puberty and hormones in adolescents with Nodding Syndrome; a pilot study. BMC Res Notes. 2014;7(1):858. doi:10.1186/1756-050 $\underline{0-7-858}$

38. Sejvar JJ, Kakooza AM, Foltz JL, et al. Clinical, neurological, and electrophysiological features of nodding syndrome in Kitgum, Uganda: an observational case series. Lancet Neurol. 2013;12(2):166-174. doi:10.1016/s1474-4422(12)7032 $1-6$

39. Spencer P. Nodding Syndrome: an epileptic disorder restricted to Africa? J Neurol Sci. 2015;357:e509. doi:10.1016/i.jns.2015.09.332

40. Spencer PS, Vandemaele K, Richer M, et al. Nodding syndrome in Mundri county, South Sudan: environmental, nutritional and infectious factors. $A f r$ Health Sci. 2013;13(2):183-204. doi:10.4314/ahs.v13i 2.2

41. Spencer PS, Mazumder R, Palmer VS, et al. Environmental, dietary and case-control study of Nodding Syndrome in Uganda: A post-measles brain disorder triggered by malnutrition? J Neurol Sci. 2016;369:191-203. doi:10.1016/i.jns.2016.08.023

42. Spencer PS, Kitara DL, Gazda SK, Winkler AS. Nodding syndrome: 2015 International Conference Report and Gulu Accord. eNeurologicalSci. 2015;3:80-83. doi:10.1016/i.ensci.2015.11.001 
43. Winkler AS, Friedrich K, König R, et al. The head nodding syndrome-clinical classification and possible causes. Epilepsia. 2008;49(12):2008-2015. doi:10.111 1/j.1528-1167.2008.01671.x

44. Winkler AS, Friedrich K, Velicheti S, et al. MRI findings in people with epilepsy and nodding syndrome in an area endemic for onchocerciasis: an observational study. Afr Health Sci. 2013;13(2). doi:1 $\underline{0.4314 / \mathrm{ahs} . v 13 \mathrm{i} 2.51}$

45. Colebunders R, Suykerbuyk P, Jacob ST, van Oijen M. Nodding syndrome, other forms of epilepsy, and the Nakalanga syndrome most likely directly or indirectly caused by Onchocerca volvulus. J Neurol Sci. 2017;372:439-440. doi:10.1016/j.jns.2016.10.008

46. Colebunders R, Post R, O’Neill S, et al. Nodding syndrome since 2012: recent progress, challenges and recommendations for future research. Trop Med Int Health. 2015;20(2):194-200. doi:10.1111/tmi.12421

47. Lee HJ, Bach JH, Chae HS, et al. Mitogen-activated protein kinase/extracellular signal-regulated kinase attenuates 3-hydroxykynurenine-induced neuronal cell death. J Neurochem. 2004;88(3):647-656. doi:10.1 111/j.1471-4159.2004.02191.x

48. Soldatos A, Nutman T, Groden C, Wahl C, Inati S, Buckler G. Evaluation and Immunomodulatory Treatment at the NIH of Children with Nodding Syndrome from Northern Uganda (S37.005). Neurology. 2015;6;84(14 Supplement).

49. Frye RE, Melnyk S, MacFabe DF. Unique acylcarnitine profiles are potential biomarkers for acquired mitochondrial disease in autism spectrum disorder. Transl Psychiatry. 2013;3:e220. doi:10.1038/t p.2012.143

50. Frye RE. Biomarkers of Abnormal Energy Metabolism in Children with Autism Spectrum Disorder. Am Chin J Med Sci. 2012;5(3):141. doi:10.71 56/v5i3p141

51. Macfabe DF. Short-chain fatty acid fermentation products of the gut microbiome: implications in autism spectrum disorders. Microb Ecol Health Dis. 2012:23. doi:10.3402/mehd.v23i0.19260

52. Musisi S, Akena D, Nakimuli-Mpungu E, Abbo C, Okello J. Neuropsychiatric perspectives on nodding syndrome in northern Uganda: a case series study and a review of the literature. Afr Health Sci. 2013;13(2):205-218. doi:10.4314/ahs.v13i2.3

53. Nyungura JL, Akim T, Lako A, Gordon A, Lejeng L, William G. Investigation into the Nodding syndrome in Witto Payam, Western Equatoria State, 2010. South Sudan Med J. 2011;4:3-6.
54. Colebunders R, Nelson Siewe FJ, Hotterbeekx A. Onchocerciasis-Associated Epilepsy, an Additional Reason for Strengthening Onchocerciasis Elimination Programs. Trends Parasitol. 2018;34(3):208-216. doi:1 0.1016/j.pt.2017.11.009

55. Colebunders R, Hendy A, van Oijen M. Nodding Syndrome in Onchocerciasis Endemic Areas. Trends Parasitol. 2016;32(8):581-583. doi:10.1016/j.pt.2016.0 $\underline{5.013}$

56. Wamala JF, Malimbo M, Tepage F, et al. Nodding Syndrome May be only the ears of the hippo. PLoS Negl Trop Dis. 2015;9(8):e0003880. doi:10.1371/journ al.pntd.0003880

57. Kitara D, Oh J, Mwaka AD. Nodding Syndrome in Uganda-a disease cluster: An epidemiological dilemma. Pak J Med Sci. 2013;11:21-23.

58. Colebunders R, Hendy A, Mokili JL, et al. Nodding syndrome and epilepsy in onchocerciasis endemic regions: comparing preliminary observations from South Sudan and the Democratic Republic of the Congo with data from Uganda. BMC Res Notes. 2016;9:182. doi:10.1186/s13104-016-1993-7

59. Colebunders R, Irani J, Post R. Nodding syndrome-we can now prevent it. Int J Infect Dis. 2016;44:61-63. doi:10.1016/i.ijid.2016.01.016

60. König R, Nassri A, Meindl M, et al. The role of Onchocerca volvulus in the development of epilepsy in a rural area of Tanzania. Parasitology. 2010;137(10):1559-1568. doi:10.1017/s003118201000 0338

61. Duke BO, Vincelette J, Moore PJ. Microfilariae in the cerebrospinal fluid, and neurological complications, during treatment of onchocerciasis with diethylcarbamazine. Tropenmed Parasitol. 1976;27:123-132.

62. World Health Organisation. Subacute sclerosing panencephalitis and measles vaccination. Global vaccine safety. Accessed July 17, 2018. http://www.wh o.int/vaccine safety/committee/topics/measles sspe/I an 2006/en/

63. Spencer PS, Schmutzhard E, Winkler AS. Nodding Syndrome in the Spotlight - Placing Recent Findings in Perspective. Trends Parasitol. 2017;33(7):490-492. doi:10.1016/j.pt.2017.05.001

64. Hotterbeekx A, Onzivua S, Menon S, Colebunders R. Histological examination of post-mortem brains of children with nodding syndrome. Ann Transl Med. 2018;6(7):134. doi:10.21037/atm.2018.02.04 
65. Payne FE, Baublis JV, Itabashi HH. Isolation of Measles Virus from Cell Cultures of Brain from a Patient with Subacute Sclerosing Panencephalitis. $N$ Engl J Med. 1969;281(11):585-589. doi:10.1056/nejm1 96909112811103

66. Britton J. Chapter 13 - Autoimmune epilepsy. In: Pittock SJ, Vincent A, eds. Handbook of Clinical Neurology. Elsevier; 2016:219-245. doi:10.1016/b97 8-0-444-63432-0.00013-X

67. Kostyukova AS. Leiomodin/tropomyosin interactions are isoform specific. Arch Biochem Biophys. 2007;465:227-230. doi:10.1016/j.abb.2007.0 $\underline{5.012}$

68. Johnson T, Nutman T, Dowell S, Nath A. Closing the Loop between Nodding Syndrome and Onchocerca Infection. Trends Parasitol. 2017;33(7):490. doi:10.1016/j.pt.2017.05.002

69. Buchmann K. 'These nodding people': Experiences of having a child with nodding syndrome in postconflict Northern Uganda. Epilepsy Behav. 2015;42:71-77. doi:10.1016/i.yebeh.2014.10.027

70. Buchmann K. 'You sit in fear': understanding perceptions of nodding syndrome in post-conflict northern Uganda. Glob Health Action. 2014;7:25069. $\underline{\mathrm{d}}$ oi:10.3402/gha.v7.25069

71. Mutamba B, Abbo C, Muron J, Idro R, Mwaka AD. Stereotypes on Nodding syndrome: responses of health workers in the affected region of northern Uganda. Afr Health Sci. 2013;13(4):986-991. doi:10.43 14/ahs.v13i4.18

72. Atim P, Ochola E, Ssendagire S, Rutebemberwa E. Health Seeking Behaviours among Caretakers of Children with Nodding Syndrome in Pader District Northern Uganda: A Mixed Methods Study. PLoS One. 2016;11(7):e0159549. doi:10.1371/journal.pone.0159 $\underline{549}$

73. Nakigudde J, Mutamba BB, Bazeyo W, Musisi S, James O. An exploration of caregiver burden for children with nodding syndrome (lucluc) in Northern Uganda. BMC Psychiatry. 2016;16:255. doi:10.1186/s1 2888-016-0955-X

74. Mutamba BB, Kane JC, de Jong JTVM, Okello J, Musisi S, Kohrt BA. Psychological treatments delivered by community health workers in lowresource government health systems: effectiveness of group interpersonal psychotherapy for caregivers of children affected by nodding syndrome in Uganda. Psychol Med. 2018;48(15):2573-2583. doi:10.1017/s00 33291718000193
75. Kitara DL, Mwaka AD, Wabinga HR, Bwangamoi PO. Pyomyositis in Nodding Syndrome (NS) patient a case report. Pan Afr Med J. 2013;16:65. doi:10.1160 4/pamj.2013.16.65.2403

76. Winkler AS, Wallner B, Friedrich K, et al. A longitudinal study on nodding syndrome-a new African epilepsy disorder. Epilepsia. 2014;55:86-93. ㄸ oi:10.1111/epi.12483

77. de Polo G, Romaniello R, Otim A, Benjamin K, Bonanni P, Borgatti R. Neurophysiological and clinical findings on Nodding Syndrome in 21 South Sudanese children and a review of the literature. Seizure. 2015;31:64-71. doi:10.1016/i.seizure.2015.0 $\underline{7.006}$

78. Kakooza-Mwesige A, Dhossche DM, Idro R, Akena D, Nalugya J, Opar BT. Catatonia in Ugandan children with nodding syndrome and effects of treatment with lorazepam: a pilot study. BMC Res Notes. 2015;8:825. doi:10.1186/s13104-015-1805-5

79. Kluxen G, Hörauf A. Ocular onchocerciasis: a key role for Wolbachia. Ophthalmologe. 2007;104(10):860-865. doi:10.1007/s00347-007-159 $\underline{4-X}$

80. Walker M, Specht S, Churcher TS, Hoerauf A, Taylor MJ, Basáñez MG. Therapeutic Efficacy and Macrofilaricidal Activity of Doxycycline for the Treatment of River Blindness. Clin Infect Dis. 2015;60(8):1199-1207. doi:10.1093/cid/ciu1152

81. Anguzu R, Akun PR, Ogwang R, et al. Setting up a clinical trial for a novel disease: a case study of the Doxycycline for the Treatment of Nodding Syndrome Trial - challenges, enablers and lessons learned. Glob Health Action. 2018;11:1431362. doi:10.1080/1654971 6.2018.1431362

82. Colebunders R, Mandro M, Mukendi D, Dolo H, Suykerbuyk P, Van Oijen M. Ivermectin Treatment in Patients With Onchocerciasis-Associated Epilepsy: Protocol of a Randomized Clinical Trial. JMIR Res Protoc. 2017;6(8):e137. doi:10.2196/resprot.7186

83. Annual Meeting of the British-PaediatricNeurology-Association, Cambridge, UK, January 11 -13, 2017. Dev Med Child Neurol. 2017;59:4-108.

84. Lagoro DK, Arony DA. Nodding syndrome (NS) and Onchocerca Volvulus (OV) in Northern Uganda. Pan Afr Med J. 2017;28:1. doi:10.11604/pamj.2017.2 $\underline{8.1 .13554}$

85. Udall DN. Recent Updates on Onchocerciasis: Diagnosis and Treatment. Clin Infect Dis. 2007;44:53-60. doi:10.1086/509325 
86. Kaiser C, Pion SDS, Boussinesq M. Case-control Studies on the Relationship between Onchocerciasis and Epilepsy: Systematic Review and Meta-analysis. PLoS Negl Trop Dis. 2013;7(3):e2147. doi:10.1371/jour nal.pntd.0002147

87. Al-Kubati AS, Mackenzie CD, Boakye D, et al. Onchocerciasis in Yemen: moving forward towards an elimination program. Int Health.

2018;10(suppl_1):i89-i96. doi:10.1093/inthealth/ihx0 $\underline{55}$

88. Sauerbrey M, Rakers LJ, Richards FO. Progress toward elimination of onchocerciasis in the Americas. Int Health. 2018;10(suppl_1):i71-i78. doi:10.1093/inth ealth/ihx039

89. Schneider-Schaulies S, Schneider-Schaulies J. Measles virus-induced immunosuppression. Curr Top Microbiol Immunol. 2009;330:243-269. doi:10.1007/97 8-3-540-70617-5 12
90. Low A, Gavriilidis G, Larke N, et al. Incidence of Opportunistic Infections and the Impact of Antiretroviral Therapy Among HIV-Infected Adults in Low- and Middle-Income Countries: A Systematic Review and Meta-analysis. Clin Infect Dis. 2016;62(12):1595-1603. doi:10.1093/cid/ciw125

91. Valdes Angues R, Suits A, Palmer VS, et al. A realtime medical cartography of epidemic disease (Nodding syndrome) using village-based lay mHealth reporters. PLoS Negl Trop Dis. 2018;12(6):e0006588. $\underline{\mathrm{d}}$ oi:10.1371/journal.pntd.0006588

92. Zikherman J, Parameswaran R, Weiss A.

Endogenous antigen tunes the responsiveness of naive B cells but not T cells. Nature. 2012;489(7414):160-164. doi:10.1038/nature11311

93. University of York. Centre for Reviews and Dissemination. PROSPERO. Accessed September 1, 2018. https://www.crd.york.ac.uk/prospero/

94. U.S. National Library of Medicine. Doxycycline for the Treatment of Nodding Syndrome - Full Text View - ClinicalTrials.gov. Accessed June 18, 2018. https://cl inicaltrials.gov/ct2/show/NCT02850913 